\title{
Isometries between spaces of weighted holomorphic functions
}

\author{
by \\ Christopher Boyd (Dublin) and Pilar Rueda (Valencia)
}

\begin{abstract}
We study isometries between spaces of weighted holomorphic functions. We show that such isometries have a canonical form determined by a group of homeomorphisms of a distinguished subset of the range and domain. A number of invariants for these isometries are determined. For specific families of weights we classify the form isometries can take.
\end{abstract}

1. Introduction. Over the past ten years the isomorphic structure of spaces of weighted holomorphic functions has been largely determined. The characterisation of weighted spaces of holomorphic functions up to isomorphism was initiated by Lusky in a series of papers [20]-[25]. In these papers he showed that if $v$ is a continuous, strictly positive, radial weight on the unit disc $\Delta$ with $v$ converging to 0 on the boundary of $\Delta$ then $\mathcal{H}_{v_{0}}(\Delta)$ is isomorphic to a subspace of $c_{0}$. Moreover, Lusky [21] gives an example of a radial weight $v$ on $\Delta$ such that $\mathcal{H}_{v_{0}}(\Delta)$ is isomorphic to a proper subspace of $c_{0}$ rather than $c_{0}$ itself. Recently Bonet and Wolf [6] have shown that if $v$ is a continuous, strictly positive weight on a bounded open subset of $\mathbb{C}^{n}$ which converges to 0 on the boundary of $U$ then $\mathcal{H}_{v_{0}}(U)$ is almost isometrically isomorphic to a subspace of $c_{0}$.

In [7] and [8] the authors undertook a detailed study of the Banach space geometry of spaces of weighted holomorphic functions $\mathcal{H}_{v}(U)$ and $\mathcal{H}_{v_{0}}(U)$. There, it was observed that the geometric structure of these spaces was determined by a distinguished subset of $U$ which we call the $v$-boundary of $U$. In this paper we see that the $v$-boundary provides us with a method of determining the isometries between weighted spaces of holomorphic functions. By isometry we shall always mean a surjective linear isometry.

Some definitions are perhaps in order. Let $U$ be a bounded open subset of $\mathbb{C}^{n}$. A continuous weight $v$ on $U$ is a bounded, strictly positive real-valued

2000 Mathematics Subject Classification: Primary 46B04, 46E15.

Key words and phrases: weighted spaces of holomorphic functions, Banach-Stone theorems. 
function on $U$. We will use $\mathcal{H}_{v}(U)$ to denote the space of all holomorphic functions $f$ on $U$ which have the property that

$$
\|f\|_{v}:=\sup _{z \in U} v(z)|f(z)|<\infty
$$

endowed with the norm $\|\cdot\|_{v}$. Consider all $f$ in $\mathcal{H}_{v}(U)$ with the property that $|f(z)| v(z)$ converges to 0 as $z$ converges to the boundary of $U$, i.e. given $\varepsilon>0$ there is a compact subset $K$ of $U$ such that $v(z)|f(z)|<\varepsilon$ for $z$ in $U \backslash K$. The set of all such functions is a subspace of $\mathcal{H}_{v}(U)$ denoted by $\mathcal{H}_{v_{0}}(U)$.

We say that the weight $v$ on a balanced domain is radial if $v(\lambda z)=v(z)$ for all $\lambda$ in $\Gamma:=\{\lambda \in \mathbb{C}:|\lambda|=1\}$, and a weight $v$ on the unit ball of $\mathbb{C}^{n}$ is unitary if it is invariant under all unitary matrices. In [7] we showed that the set of extreme points of the unit ball of $\mathcal{H}_{v_{0}}(U)^{\prime}$ is contained in $\left\{\lambda v(z) \delta_{z}: z \in U, \lambda \in \Gamma\right\}$. The $v$-boundary of $U$ is defined as the set of all $z \in U$ such that $v(z) \delta_{z}$ is an extreme point of the unit ball of $\mathcal{H}_{v_{0}}(U)^{\prime}$. Note that $v(x) \delta_{x}$ is an extreme point of the unit ball of $\mathcal{H}_{v_{0}}(U)^{\prime}$ if and only if $\lambda v(x) \delta_{x}$ is an extreme point for every $\lambda$ in $\Gamma$. We use $\mathcal{B}_{v}(U)$ to denote the $v$-boundary.

It is shown in [7] that $\mathcal{B}_{v}(U)$ is radial when $v$ is radial and unitary when $v$ is unitary. Furthermore, the mapping $\mu: U \rightarrow\left(\mathcal{H}_{v_{0}}(U)^{\prime}, \sigma\left(\mathcal{H}_{v_{0}}(U)^{\prime}, \mathcal{H}_{v_{0}}(U)\right)\right)$, $\mu(z)=v(z) \delta_{z}$ is a homeomorphism onto its range that allows us to show that $\mathcal{B}_{v}(U)$ is a $G_{\delta}$ subset of $U$. Here $\sigma\left(\mathcal{H}_{v_{0}}(U)^{\prime}, \mathcal{H}_{v_{0}}(U)\right)$ denotes the weak* topology on $\mathcal{H}_{v_{0}}(U)^{\prime}$. We say that a weight $v$ on $U$ is complete if $\mathcal{B}_{v}(U)=U$. A sufficient criterion for a unitary weight $v$ on the unit ball of $\mathbb{C}^{n}$ to be complete is given in [8, Proposition 18].

In the first section we give three Banach-Stone theorems for spaces of weighted holomorphic functions on bounded subsets of $\mathbb{C}^{n}$. We show that the isometries from $\mathcal{H}_{v_{0}}(U)$ onto $\mathcal{H}_{w_{0}}(V)$ are determined by a homeomorphism $\phi$ of $\mathcal{B}_{w}(V)$ onto $\mathcal{B}_{v}(U)$. If $U$ and $V$ are strictly convex with $\mathcal{B}_{v}(U)$ non-empty we shall see that $\phi$ can be extended to a homeomorphism of $V$ onto $U$ and that the isometries of $\mathcal{H}_{v_{0}}(U)$ determine the isometries of $\mathcal{H}_{v}(U)$. In [7] we proved that $\mathcal{H}_{v_{0}}(U)$ has trivial centraliser when $v$ is radial. This, along with a result of Jarosz [16], allows us to characterise the isometries of spaces of vector-valued weighted spaces of holomorphic functions. Given a weight $v$ we will see that the homeomorphisms $\phi$ which determine an isometry of $\mathcal{H}_{v_{0}}(U)$ form a group. This group is called the isometry group of the weight. Isometry groups can be calculated for specific families of weights with the help of potential theory. Using a result of Henri Cartan we characterise the isometries of $\mathcal{H}_{\left(v_{1} \times v_{2}\right)_{0}}\left(U_{1} \times U_{2}\right)$. We characterise those weights on the unit disc $\Delta$ whose isometry group contains all rotations, and completely determine the weights on the unit disc which have isometry group equal to $\operatorname{Aut}(\Delta)$. 
2. Three Banach-Stone theorems. In this section we shall characterise the isometries $T$ of $\mathcal{H}_{v_{0}}(U)$ onto $\mathcal{H}_{w_{0}}(V)$.

We note that it is relatively easy to construct "different" weights which give isometric spaces of holomorphic functions. Let $U$ be a bounded open subset of $\mathbb{C}^{n}$ and consider $g \in \mathcal{H}^{\infty}(U)$. Then $e^{\operatorname{Re} g}$ is a bounded strictly positive harmonic function on $U$. Let $v$ be a continuous strictly positive weight on $U$ which converges to 0 on the boundary of $U$ and define $w(z)$ by $w(z):=e^{-\operatorname{Re} g(z)} v(z)$. Then $w$ is also a continuous strictly positive weight on $U$ which converges to 0 on the boundary of $U$. Furthermore, the multiplication operator $M_{e^{g}}$ defined by $\left(M_{e^{g}}(f)\right)(z)=f(z) e^{g(z)}$ is an isometric isomorphism of $\mathcal{H}_{v_{0}}(U)$ onto $\mathcal{H}_{w_{0}}(U)$. We note that if $U$ is balanced, $v$ is radial and $g$ is non-constant then $w$ will be a non-radial weight on $U$ with the property that $\mathcal{H}_{v_{0}}(U)$ is isometrically isomorphic to $\mathcal{H}_{w_{0}}(U)$.

Given a Banach space $E$ we use Ext $B_{E}$ to denote the set of extreme points of the unit ball of $E$.

Theorem 1 (A first Banach-Stone theorem). Let $U, V$ be bounded open subsets of $\mathbb{C}^{n_{1}}$ and $\mathbb{C}^{n_{2}}$ respectively. Let $v: U \rightarrow \mathbb{R}$ and $w: V \rightarrow \mathbb{R}$ be continuous strictly positive weights converging to 0 on their respective boundaries. If $T: \mathcal{H}_{v_{0}}(U) \rightarrow \mathcal{H}_{w_{0}}(V)$ is an isometric isomorphism, then there is a homeomorphism $\phi: \mathcal{B}_{w}(V) \rightarrow \mathcal{B}_{v}(U)$ and $h_{\phi} \in \mathcal{H}_{w_{0}}(V)$ such that

$$
T(f)(z)=h_{\phi}(z) f \circ \phi(z) \quad \text { for all } f \in \mathcal{H}_{v_{0}}(U), z \in \mathcal{B}_{w}(V) .
$$

Moreover,

$$
\left|h_{\phi}\right|=\frac{v \circ \phi}{w} \quad \text { on } \mathcal{B}_{w}(V) .
$$

Further, if $\stackrel{\mathcal{B}}{w}_{w}(V)$ is non-empty then $\phi: \stackrel{\mathcal{B}}{w}_{w}(V) \rightarrow \stackrel{\mathcal{B}}{v}_{v}(U)$ is a biholomorphic mapping.

Proof. For $z_{1}, z_{2}$ in $\overline{\mathcal{B}_{v}(U)}$ or $\overline{\mathcal{B}_{w}(V)}$ define the relation $\sim$ by $z_{1} \sim z_{2}$ if $z_{1}=z_{2}$ or if $z_{1}, z_{2}$ are both in $\partial U$ or in $\partial V$. Let $q_{1}: \overline{\mathcal{B}_{v}(U)} \rightarrow \overline{\mathcal{B}_{v}(U)} / \sim$ and $q_{2}: \overline{\mathcal{B}_{w}(V)} \rightarrow \overline{\mathcal{B}_{w}(V)} / \sim$ be the quotient maps. Given $z$ in $\overline{\mathcal{B}_{v}(U)}$ or $\overline{\mathcal{B}_{w}(V)}$ we shall use $\widetilde{z}$ to denote $q_{1}(z)$ or $q_{2}(z)$ depending on whether $z$ belongs to $\overline{\mathcal{B}_{v}(U)}$ or $\overline{\mathcal{B}_{w}(V)}$.

Consider the transpose $T^{*}: \mathcal{H}_{w_{0}}(V)^{\prime} \rightarrow \mathcal{H}_{v_{0}}(U)^{\prime}$ of $T$. Since $T$ is an isometric isomorphism, $T^{*}$ is a weak ${ }^{*}$-to-weak ${ }^{*}$ homeomorphism and maps $\overline{\operatorname{Ext}\left(B_{\mathcal{H}_{w_{0}}(V)^{\prime}}\right.} \sigma^{*}$ onto $\overline{\operatorname{Ext}\left(B_{\mathcal{H}_{v_{0}}(U)^{\prime}}\right)^{*}}$. Then, by [7, Lemma 10], for each $z \in \overline{\mathcal{B}_{w}(V)}$ there exist $\alpha(z) \in \Gamma$ and $\phi(z) \in \overline{\mathcal{B}_{v}(U)}$ such that

$$
T^{*}\left(w(z) \delta_{z}\right)=\alpha(z) v(\phi(z)) \delta_{\phi(z)} .
$$

Define the map $\widetilde{\phi}: q_{2}\left(\overline{\mathcal{B}_{w}(V)}\right) \rightarrow q_{1}\left(\overline{\mathcal{B}_{v}(U)}\right)$ by $\widetilde{\phi}(\widetilde{z}):=\widetilde{\phi(z)}$. Let us see that $\widetilde{\phi}$ is well defined. Suppose $\widetilde{z}_{1}=\widetilde{z}_{2}$. 
If $z_{1}=z_{2}$ then $\alpha\left(z_{1}\right) v\left(\phi\left(z_{1}\right)\right) \delta_{\phi\left(z_{1}\right)}=\alpha\left(z_{2}\right) v\left(\phi\left(z_{2}\right)\right) \delta_{\phi\left(z_{2}\right)}$, and by [7, Lemma 9], $\widetilde{\phi\left(z_{1}\right)}=\widetilde{\phi\left(z_{2}\right)}$.

On the other hand, if $z_{1}, z_{2} \in \partial V$ then $w\left(z_{1}\right) \delta_{z_{1}}=w\left(z_{2}\right) \delta_{z_{2}}=0$. Since $T^{*}$ is linear,

$$
\alpha\left(z_{1}\right) v\left(\phi\left(z_{1}\right)\right) \delta_{\phi\left(z_{1}\right)}=\alpha\left(z_{2}\right) v\left(\phi\left(z_{2}\right)\right) \delta_{\phi\left(z_{2}\right)}=0,
$$

and by [7, Lemma 9], $\phi\left(z_{1}\right), \phi\left(z_{2}\right) \in \partial U$.

Let us now prove that $\widetilde{\phi}$ is a homeomorphism. To prove that $\widetilde{\phi}$ is injective suppose that $\widetilde{\phi\left(z_{1}\right)}=\widetilde{\phi\left(z_{2}\right)}$.

If $\phi\left(z_{1}\right)=\phi\left(z_{2}\right)$ then

$\frac{1}{\alpha\left(z_{1}\right)} T^{*}\left(w\left(z_{1}\right) \delta_{z_{1}}\right)=v\left(\phi\left(z_{1}\right)\right) \delta_{\phi\left(z_{1}\right)}=v\left(\phi\left(z_{2}\right)\right) \delta_{\phi\left(z_{2}\right)}=\frac{1}{\alpha\left(z_{2}\right)} T^{*}\left(w\left(z_{2}\right) \delta_{z_{2}}\right)$.

Since $T^{*}$ is injective, $\frac{1}{\alpha\left(z_{1}\right)} w\left(z_{1}\right) \delta_{z_{1}}=\frac{1}{\alpha\left(z_{2}\right)} w\left(z_{2}\right) \delta_{z_{1}}$, and by [7, Lemma 9] we have $\widetilde{z}_{1}=\widetilde{z}_{2}$.

If $\phi\left(z_{1}\right), \phi\left(z_{2}\right) \in \partial U$ then $\alpha\left(z_{1}\right) v\left(\phi\left(z_{1}\right)\right) \delta_{\phi\left(z_{1}\right)}=\alpha\left(z_{2}\right) v\left(\phi\left(z_{2}\right)\right) \delta_{\phi\left(z_{2}\right)}=0$. Since $\left(T^{*}\right)^{-1}$ is linear, $w\left(z_{1}\right) \delta_{z_{1}}=w\left(z_{2}\right) \delta_{z_{2}}=0$, and by [7, Lemma 9] we have $z_{1}, z_{2} \in \partial V$.

We now prove that $\widetilde{\phi}$ is surjective. Take $z_{2} \in \overline{\mathcal{B}_{v}(U)}$. Then

$$
\left(T^{*}\right)^{-1}\left(v\left(z_{2}\right) \delta_{z_{2}}\right)=\beta\left(z_{2}\right) w\left(\psi\left(z_{2}\right)\right) \delta_{\psi\left(z_{2}\right)}
$$

for some $\beta\left(z_{2}\right) \in \Gamma$ and $\psi\left(z_{2}\right) \in \overline{\mathcal{B}_{v}(U)}$. Therefore

$$
\alpha\left(\psi\left(z_{2}\right)\right) v\left(\phi\left(\psi\left(z_{2}\right)\right)\right) \delta_{\phi\left(\psi\left(z_{2}\right)\right)}=T^{*}\left(w\left(\psi\left(z_{2}\right)\right) \delta_{\psi\left(z_{2}\right)}\right)=\frac{1}{\beta\left(z_{2}\right)} v\left(z_{2}\right) \delta_{z_{2}} .
$$

Hence, by [7, Lemma 9], either $z_{2}=\phi\left(\psi\left(z_{2}\right)\right)$, or $z_{2}$ and $\phi\left(\psi\left(z_{2}\right)\right)$ are in $\partial U$. In either case, $\widetilde{z}_{2}=\widetilde{\phi}\left(\widetilde{\psi\left(z_{2}\right)}\right)$.

Since $\overline{\mathcal{B}_{w}(V)} / \sim$ is metrisable, to prove that $\widetilde{\phi}$ is continuous we need only consider a sequence $\left(\widetilde{z}_{n}\right)_{n}$ converging to $\widetilde{z}_{0}$ in $q_{2}\left(\overline{\mathcal{B}_{w}(V)}\right)$. There are two possibilities:

CASE 1: There exists a subsequence $\left(z_{n_{k}}\right)_{k}$ in $\mathcal{B}_{w}(V)$ converging to $z_{0}$ in $\overline{\mathcal{B}_{w}(V)}$. In that case, since $\left|\alpha\left(z_{n_{k}}\right)\right|=1$ and $\phi\left(z_{n_{k}}\right)$ is in the compact set $\overline{\mathcal{B}_{v}(U)}$ for all $k$, we can assume without loss of generality that $\left(\alpha\left(z_{n_{k}}\right)\right)_{k}$ converges to some $\alpha_{0}$, and $\left(\phi\left(z_{n_{k}}\right)\right)_{k}$ converges to some $u_{0} \in \overline{\mathcal{B}_{v}(U)}$. Then $T^{*}\left(w\left(z_{n_{k}}\right) \delta_{z_{n_{k}}}\right)=\alpha\left(z_{n_{k}}\right) v\left(\phi\left(z_{n_{k}}\right)\right) \delta_{\phi\left(z_{n_{k}}\right)}$ converges weak* to $T^{*}\left(w\left(z_{0}\right) \delta_{z_{0}}\right)=$ $\alpha\left(z_{0}\right) v\left(\phi\left(z_{0}\right)\right) \delta_{\phi\left(z_{0}\right)}$ and to $\alpha_{0} v\left(u_{0}\right) \delta_{u_{0}}$. By [7, Lemma 9] we conclude that $\widetilde{u}_{0}=\widetilde{\phi}\left(\widetilde{z}_{0}\right)$ and so $\widetilde{\phi}\left(\widetilde{z}_{n_{k}}\right)$ converges to $\widetilde{\phi}\left(\widetilde{z}_{0}\right)$.

CASE 2: There exists a subsequence $\left(z_{n_{k}}\right)_{k}$ in $\partial V$. Then $\widetilde{z}_{n_{k}}=\widetilde{z}_{0}$ and hence $\widetilde{\phi}\left(\widetilde{z}_{n_{k}}\right)_{k}$ trivially converges to $\widetilde{\phi}\left(\widetilde{z_{0}}\right)$. 
Since $q_{2}\left(\overline{\left.\mathcal{B}_{w}(V)\right)}\right.$ is compact, we conclude that $\widetilde{\phi}$ is a homeomorphism.

As $T$ is an isometric isomorphism, it follows that $T^{*} \operatorname{maps} \operatorname{Ext}\left(B_{\mathcal{H}_{w_{0}}(V)^{\prime}}\right)$ onto $\operatorname{Ext}\left(B_{\mathcal{H}_{v_{0}}(U)^{\prime}}\right)$ and therefore $\widetilde{\phi}\left(q_{2}\left(\mathcal{B}_{w}(V)\right)\right)=q_{1}\left(\mathcal{B}_{v}(U)\right)$. Moreover, $\left.\widetilde{\phi}\right|_{q_{2}\left(\mathcal{B}_{w}(V)\right)}$ is an open map. To see this, let $A$ be open in $q_{2}\left(\mathcal{B}_{w}(V)\right)$. Then there exists $B$ open in $\overline{\mathcal{B}_{w}(V)}$ such that $q_{2}(B) \cap q_{2}\left(\mathcal{B}_{w}(V)\right)=A$. Hence

$$
\begin{aligned}
\widetilde{\phi}(A) & =\widetilde{\phi}\left(q_{2}(B) \cap q_{2}\left(\mathcal{B}_{w}(V)\right)\right)=\widetilde{\phi}\left(q_{2}(B)\right) \cap \widetilde{\phi}\left(q_{2}\left(\mathcal{B}_{w}(V)\right)\right) \\
& =\widetilde{\phi}\left(q_{2}(B)\right) \cap q_{1}\left(\mathcal{B}_{v}(U)\right),
\end{aligned}
$$

where the second equality follows from the fact that $\widetilde{\phi}$ is injective.

So $\left.\widetilde{\phi}\right|_{q_{2}\left(\mathcal{B}_{w}(V)\right)}: q_{2}\left(\mathcal{B}_{w}(V)\right) \rightarrow q_{1}\left(\mathcal{B}_{v}(U)\right)$ is a homeomorphism. Thus

$$
\phi=\left.\left(\left.q_{1}\right|_{\mathcal{B}_{v}(U)}\right)^{-1} \circ \widetilde{\phi} \circ q_{2}\right|_{\mathcal{B}_{w}(V)}
$$

is a homeomorphism. As $T^{*}\left(w(z) \delta_{z}\right)=\alpha(z) v(\phi(z)) \delta_{\phi(z)}$ we have

$$
T(f)(z)=\alpha(z) \frac{v \circ \phi(z)}{w(z)} f \circ \phi(z)
$$

for all $z \in \overline{\mathcal{B}_{w}(V)}$ and all $f \in \mathcal{H}_{v_{0}}(U)$. Applying this equality to the constant map $f(z) \equiv 1$ for all $z \in V$, we get

$$
\alpha(z) \frac{v(\phi(z))}{w(z)}=T(1)(z) \quad \text { for } z \in \mathcal{B}_{w}(V),
$$

and so $h_{\phi}(z):=T(1)(z)$ belongs to $\mathcal{H}_{w_{0}}(V)$. Suppose $\dot{\mathcal{B}}_{v}(U)$ is non-empty. For each linear $l: \mathbb{C}^{n} \rightarrow \mathbb{C}$ we find that $l \circ \phi(z)=h_{\phi}(z)^{-1} T(l)(z)$ is holomorphic, which implies that $\phi(z)$ is holomorphic.

The above theorem may also be deduced from [2, Theorem 4.1].

When $U$ is balanced and $v$ is radial, the isometries of $\mathcal{H}_{v_{0}}(U)$ determine the isometries of $\mathcal{H}_{v}(U)$ as the following theorem illustrates.

Theorem 2 (A second Banach-Stone theorem). Let $U$ be a balanced bounded open subset of $\mathbb{C}^{n}$, and let $v$ be a continuous strictly positive radial weight converging to 0 on the boundary of $U$. If $T: \mathcal{H}_{v}(U) \rightarrow \mathcal{H}_{v}(U)$ is a isometric isomorphism, then there is a homeomorphism $\phi: \mathcal{B}_{v}(U) \rightarrow \mathcal{B}_{v}(U)$ and $h_{\phi} \in \mathcal{H}_{v_{0}}(U)$ such that

$$
T(f)(z)=h_{\phi}(z) f \circ \phi(z) \quad \text { for all } f \in \mathcal{H}_{v}(U), z \in \mathcal{B}_{v}(U) .
$$

Moreover,

$$
\left|h_{\phi}(z)\right|=\frac{v \circ \phi(z)}{v(z)} \quad \text { on } \mathcal{B}_{v}(U)
$$

$h_{\phi}$ coincides with the function $h_{\phi}$ obtained in Theorem 1 and $T$ maps $\mathcal{H}_{v_{0}}(U)$ onto $\mathcal{H}_{v_{0}}(U)$. Further, if $\dot{\mathcal{B}}_{v}(U)$ is non-empty, then $\phi: \dot{\mathcal{B}}_{v}(U) \rightarrow \dot{\mathcal{B}}(U)$ is an analytic automorphism. 
Proof. We observed in the proof of [7, Proposition 14] (see also [15, Examples III.1.4]) that $\mathcal{H}_{v_{0}}(U)$ is an M-ideal in $\mathcal{H}_{v}(U)$. Hence, by [14, Proposition 4.2], every isometry of $\mathcal{H}_{v}(U)$ is the bitranspose of an isometry of $\mathcal{H}_{v_{0}}(U)$ and hence the transpose of an isometry of $\mathcal{H}_{v_{0}}(U)^{\prime}$. But we have seen that isometries $T^{*}$ of $\mathcal{H}_{v_{0}}(U)^{\prime}$ all have the form

$$
T^{*}\left(v(z) \delta_{z}\right)=\alpha(z) v(\phi(z)) \delta_{\phi(z)}
$$

for $z \in \mathcal{B}_{v}(U)$ where $\phi$ is a homeomorphism of $\mathcal{B}_{v}(U)$. From this observation we deduce that every isometry of $\mathcal{H}_{v}(U)$ has the form $T(f)(z)=h_{\phi}(z) f \circ$ $\phi(z)$ for $h_{\phi}: U \rightarrow \mathbb{C}$. Finally, when we take $f \equiv 1$, as $T$ maps $\mathcal{H}_{v_{0}}(U)$ onto $\mathcal{H}_{v_{0}}(U)$ we see that $h_{\phi} \in \mathcal{H}_{v_{0}}(U)$. From the proof of Theorem 1 it follows that $\phi$ is an automorphism of $\stackrel{\circ}{\mathcal{B}}_{v}(U)$ when $\dot{\mathcal{B}}_{v}(U)$ is non-empty.

We will show that for certain weights $v$ the homeomorphism $\phi: \mathcal{B}_{w}(V) \rightarrow$ $\mathcal{B}_{v}(U)$ can be extended to an automorphism of $V$ onto $U$.

TheOREM 3. Let $U$ and $V$ be bounded, strictly convex open subsets of $\mathbb{C}^{n}$. Let $v: U \rightarrow \mathbb{R}$ and $w: V \rightarrow \mathbb{R}$ be continuous strictly positive weights which converge to 0 on their respective boundaries. Suppose in addition that $\stackrel{\mathcal{B}}{w}_{w}(V)$ is non-empty. If $T: \mathcal{H}_{v_{0}}(U) \rightarrow \mathcal{H}_{w_{0}}(V)$ is an isometric isomorphism, then there is a biholomorphic mapping $\phi: V \rightarrow U$ and $h_{\phi} \in \mathcal{H}_{w_{0}}(V)$ such that

$$
T(f)(z)=h_{\phi}(z) f \circ \phi(z) \quad \text { for all } f \in \mathcal{H}_{v_{0}}(U), z \in V .
$$

Moreover,

$$
\left|h_{\phi}(z)\right|=\frac{v \circ \phi(z)}{w(z)} \quad \text { for all } z \in \mathcal{B}_{v}(U) .
$$

Proof. Theorem 1 ensures the existence of a biholomorphic mapping $\phi: \dot{\mathcal{B}}_{w}(V) \rightarrow \check{\mathcal{B}}_{v}(U)$ and $h_{\phi} \in \mathcal{H}_{w_{0}}(V)$ such that

$$
T(f)(z)=h_{\phi}(z) f \circ \phi(z) \quad \text { for all } f \in \mathcal{H}_{v_{0}}(U), z \in \dot{\mathcal{B}}_{w}(V) .
$$

We will show that $\phi$ extends to a biholomorphic mapping from $V$ onto $U$. Without loss of generality we may suppose that $\sup _{z \in U} v(z) \leq 1$. Let $l: \mathbb{C}^{n} \rightarrow \mathbb{C}$ be linear. By $(*)$ we have

$$
l \circ \phi(z)=h_{\phi}(z)^{-1} T(l)(z) \quad \text { for all } z \in \dot{\mathcal{B}}_{w}(V) .
$$

Since the right-hand side of this equation is defined for all $z$ in $V \backslash\{z$ : $\left.h_{\phi}(z)=0\right\}$ and all linear $l: \mathbb{C}^{n} \rightarrow \mathbb{C}$, we see that $\phi$ has a holomorphic extension to $V \backslash\left\{z: h_{\phi}(z)=0\right\}$, which we denote by $\widetilde{\phi}$. Let $k$ be a positive integer and $l$ be a linear functional on $\mathbb{C}^{n}$ with $\sup _{z \in U}|l(z)| \leq 1$. As $\left(l^{k}\right)_{k \in \mathbb{N}} \subset \mathcal{H}_{v_{0}}(U)$ and each $l^{k}$ has norm at most 1 , we see that $\left\|T\left(l^{k}\right)\right\|_{w} \leq 1$. For $z \in \hat{\mathcal{B}}_{w}(V)$ we have

$$
T\left(l^{k}\right)(z)=h_{\phi}(z)(l \circ \phi(z))^{k}=h_{\phi}(z)(l \circ \widetilde{\phi}(z))^{k} .
$$


Since $\left\{z: h_{\phi}(z)=0\right\}$ is an analytic subset of $V,[27$, Proposition 4.1] implies that $V \backslash\left\{z: h_{\phi}(z)=0\right\}$ is connected.

The principle of analytic continuation now implies that

$$
T\left(l^{k}\right)(z)=h_{\phi}(z)(l \circ \widetilde{\phi}(z))^{k} \quad \text { for all } z \in V \backslash\left\{z: h_{\phi}(z)=0\right\} .
$$

Taking $k$ th roots and letting $k \rightarrow \infty$ we see that $\widetilde{\phi}\left(V \backslash\left\{z: h_{\phi}(z)=0\right\}\right)$ is bounded. By Riemann's continuation theorem [27, Proposition 4.2], $\widetilde{\phi}$ has an analytic extension to $V$, which we also denote by $\widetilde{\phi}$.

We claim that $\widetilde{\phi}$ maps $V$ into $U$. First we show that $\widetilde{\phi}(V) \subset \bar{U}$. Suppose this is not the case. Then we can choose a continuous linear functional, $l$, on $U$ with norm at most 1 so that $|l(\widetilde{\phi}(z))|>1$ for some $z \in V$. Continuity allows us to suppose in addition that $h_{\phi}(z) \neq 0$. We have

$$
T\left(l^{k}\right)(z)=h_{\phi}(z) l(\widetilde{\phi}(z))^{k},
$$

and thus

$$
\left|T\left(l^{k}\right)(z)\right| w(z)=\left|h_{\phi}(z)\right| w(z)|l(\widetilde{\phi}(z))|^{k},
$$

for all $k \in \mathbb{N}$ and all $z \in V$. Taking $k$ th roots and letting $k$ tend to infinity gives a contradiction and therefore $|l(\widetilde{\phi}(z))| \leq 1$ for all linear $l$ with $\|l\|_{U} \leq 1$. Hence $\widetilde{\phi}(V) \subseteq \bar{U}$. An application of Thorp and Whitley's strong maximum modulus theorem [32, Theorem 3.1] implies that we must have $\widetilde{\phi}(V) \subseteq U$.

Let $\widetilde{\psi}$ denote the extension of $\phi^{-1}$ to $U$ obtained in the same way as above. By the principle of analytic continuation we have

$$
\widetilde{\psi} \circ \widetilde{\phi}=\operatorname{Id}_{V} \text { and } \widetilde{\phi} \circ \widetilde{\psi}=\operatorname{Id}_{U} .
$$

Hence $\widetilde{\phi}$ is a biholomorphic mapping of $V$ onto $U$. Using the principle of analytic continuation once again we get $T(f)(z)=h_{\phi}(z) f \circ \widetilde{\phi}(z)$ for all $f \in \mathcal{H}_{v_{0}}(U)$ and $z \in V$.

When $n=1$, the principle of analytic continuation may be replaced by the identity principle, and the condition that the $v$-boundary of $U$ has nonempty interior can be replaced by the weaker condition that the $v$-boundary of $U$ has an accumulation point in $U$. This holds in particular when $v$ and $w$ are radial. In such cases we get

TheOREM 4. Let $v, w: \Delta \rightarrow \mathbb{R}$ be continuous strictly positive weights which converge to zero on the boundary of $\Delta$ and such that $\mathcal{B}_{v}(\Delta)$ and $\mathcal{B}_{w}(\Delta)$ have an accumulation point in $\Delta$. If $T: \mathcal{H}_{v_{0}}(\Delta) \rightarrow \mathcal{H}_{w_{0}}(\Delta)$ is an isometric isomorphism, then there is an automorphism $\phi$ of $\Delta$ and $h_{\phi} \in \mathcal{H}_{w_{0}}(\Delta)$ such that

$$
T(f)(z)=h_{\phi}(z) f \circ \phi(z) \quad \text { for all } f \in \mathcal{H}_{v_{0}}(\Delta), z \in \Delta .
$$

In particular, we have 
Corollary 5. Let $v, w: \Delta \rightarrow \mathbb{R}$ be continuous strictly positive radial weights which decrease to zero on the boundary of $\Delta$. If $T: \mathcal{H}_{v_{0}}(\Delta) \rightarrow$ $\mathcal{H}_{w_{0}}(\Delta)$ is an isometric isomorphism, then there is an automorphism $\phi$ of $\Delta$ and $h_{\phi} \in \mathcal{H}_{w_{0}}(\Delta)$ such that

$$
T(f)(z)=h_{\phi}(z) f \circ \phi(z) \quad \text { for all } f \in \mathcal{H}_{v_{0}}(\Delta), z \in \Delta .
$$

Further, if $T: \mathcal{H}_{v}(\Delta) \rightarrow \mathcal{H}_{v}(\Delta)$ is an isometry then $T$ is also of the above form.

The results in this section illustrate a fundamental difference between the isomorphic and isometric theory of spaces of weighted holomorphic functions. [21, Lemma 3.2] shows that the isomorphic structure of $\mathcal{H}_{v_{0}}(\Delta)$, when $v$ is radial, is determined by how $v$ behaves as it approaches the boundary of $\Delta$. This section shows that the isometric structure is determined by how $v$ behaves on the entire $v$-boundary.

3. A vector-valued Banach-Stone theorem. In this section we present a vector-valued Banach-Stone theorem. First we recall some definitions from [3] and theorems from [7].

Definition 6. Let $E$ be a Banach space and $T: E \rightarrow E$ be a continuous linear operator. Then $T$ is a multiplier if every extreme point of the unit ball of $E^{\prime}$ is an eigenvector of $T^{*}$. That is,

$$
T^{*}(e)=a_{T}(e) e
$$

for some real or complex number $a_{T}(e)$ and every $e \in \operatorname{Ext} B_{E^{\prime}}$. We let $\operatorname{Mult}(E)$ denote the set of all multipliers on $E$.

Definition 7. Let $E$ be a Banach space. The centraliser of $E, Z(E)$, is the set of all $T \in \operatorname{Mult}(E)$ for which there is $S$ in $\operatorname{Mult}(E)$ with $S^{*}(e)=$ $\overline{a_{T}(e)} e$ for all $e \in \operatorname{Ext} B_{E^{\prime}}$.

We say that $Z(E)$ is trivial if $Z(E)=\mathbb{K}$.Id $(\mathbb{K}=\mathbb{R}$ or $\mathbb{C}$ depending on whether $E$ is a real or complex Banach space).

Let $E$ and $F$ be Banach spaces and $u$ be an element of the tensor product $E \otimes F$. We define the injective norm of $u$ as

$$
\|u\|_{\epsilon}=\sup \left\{\left|\sum_{i=1}^{k} \phi\left(x_{i}\right) \psi\left(y_{i}\right)\right|: u=\sum_{i=1}^{k} x_{i} \otimes y_{i}, \phi \in B_{E^{\prime}}, \psi \in B_{F^{\prime}}\right\} .
$$

We shall use $E \otimes_{\epsilon} F$ to denote $E \otimes F$ endowed with the injective norm and $E \widehat{\otimes}_{\epsilon} F$ to denote the completion of $E \otimes_{\epsilon} F$ with respect to this norm.

Given Banach spaces $E$ and $F$ we let $E \epsilon F$ denote the space of all linear maps from $E^{\prime}$ into $F$ which are continuous for the compact-open topology on $E^{\prime}$ and the norm topology on $F$. We endow this space with the topology 
of uniform convergence over the unit ball of $E^{\prime}$. When $E$ or $F$ have the approximation property then $E \widehat{\otimes}_{\epsilon} F=E \epsilon F$.

In [7] we proved that if $U$ is a balanced bounded open subset of $\mathbb{C}^{n}$ and $v$ is a radial, strictly positive, continuous weight on $U$ which converges to 0 on the boundary of $U$, then $\mathcal{H}_{v_{0}}(U)$ has trivial centraliser. This result is "central" to the following result.

THEOREM 8. Let $U$ be a balanced bounded open subset of $\mathbb{C}^{n}$, and let $v$ be a continuous strictly positive radial weight on $U$ which converges to 0 on the boundary of $U$ such that $\dot{\mathcal{B}}_{v}(U)$ is non-empty. Let $E$ be a Banach space such that $E^{\prime}$ is rotund. If $T: \mathcal{H}_{v_{0}}(U ; E) \rightarrow \mathcal{H}_{v_{0}}(U ; E)$ is an isometric isomorphism, then there is an automorphism $\phi: \dot{\mathcal{B}}_{v}(U) \rightarrow \dot{\mathcal{B}}_{v}(U), h_{\phi} \in$ $\mathcal{H}_{v_{0}}(U)$ and an isometry $S: E \rightarrow E$ such that

$$
T(f)(z)=h_{\phi}(z) S(f \circ \phi(z)) \quad \text { for all } f \in \mathcal{H}_{v_{0}}(U ; E), z \in \dot{\mathcal{B}}_{v}(U) .
$$

Moreover,

$$
\left|h_{\phi}(z)\right|=\frac{v \circ \phi(z)}{v(z)} \quad \text { for all } z \text { in } \dot{\mathcal{B}}_{v}(U) .
$$

Proof. If $T$ is an isometry of $\mathcal{H}_{v_{0}}(U ; E)$ it is also an isometry of the underlying real space. By [8, Proposition 18] (see also [4]), $\mathcal{H}_{v_{0}}(U)^{\prime}$ and hence $\mathcal{H}_{v_{0}}(U)$ has the approximation property. Therefore, $T$ is a real isometry of

$$
\mathcal{H}_{v_{0}}(U ; E)=\mathcal{H}_{v_{0}}(U) \epsilon E=\mathcal{H}_{v_{0}}(U) \widehat{\otimes}_{\epsilon} E .
$$

By [7, Proposition 24], $\mathcal{H}_{v_{0}}(U)$ has trivial centraliser. It therefore follows from [16, Theorem 3] that $T$ has one of the following two forms:

(a) There is a Banach space $Z$ so that $\mathcal{H}_{v_{0}}(U)$ is isometrically isomorphic to $Z \widehat{\otimes}_{\epsilon} E$ and

$$
T(z \otimes h \otimes k)=z \otimes k \otimes h \quad \text { for all } z \in Z, h, k \in E .
$$

(b) There are (real) isometries $T_{1}$ of $\mathcal{H}_{v_{0}}(U)$ and $T_{2}$ of $E$ such that

$$
T(f \otimes y)=T_{1}(f) \otimes T_{2}(y) \quad \text { for all } f \in \mathcal{H}_{v_{0}}(U), y \in E .
$$

Suppose that $T$ is of the form given in (a). Then $\mathcal{H}_{v_{0}}(U)=Z \widehat{\otimes}_{\epsilon} E$. Since $E^{\prime}$ is rotund, every point of the unit sphere of $E^{\prime}$ is an extreme point. Fix $z^{\prime} \in \operatorname{Ext} B_{Z^{\prime}}$ and consider the set $\left\{z^{\prime} \otimes y: y \in E^{\prime},\|y\|=1\right\}$. It follows from [31, Theorem 1.1] that $\operatorname{Ext} B_{\mathcal{H}_{v_{0}}(U)^{\prime}}=\operatorname{Ext} B_{Z^{\prime}} \otimes S_{E^{\prime}}$ where $S_{E^{\prime}}$ is the unit sphere of $E^{\prime}$. Hence there is $V \subset U$ such that $\left\{z^{\prime} \otimes y: y \in E^{\prime},\|y\|=1\right\}=$ $\left\{\lambda v(z) \delta_{z}: z \in V, \lambda \in \Gamma\right\}$. The mapping from $E^{\prime}$ onto $\left\langle z^{\prime}\right\rangle \otimes E^{\prime}$ sending $y$ to $z^{\prime} \otimes y$ is an isometric isomorphism of $E^{\prime}$ onto the subspace $\left\langle z^{\prime}\right\rangle \otimes E^{\prime}$ of $\mathcal{H}_{v_{0}}(U)^{\prime}$. Since $E^{\prime}$ is rotund, $\left\langle z^{\prime}\right\rangle \otimes E^{\prime}$ is equal to $\left\{\lambda v(z) \delta_{z}: z \in V, \lambda \in \mathbb{C}\right\}$. But $\left\{\lambda v(z) \delta_{z}: z \in V, \lambda \in \mathbb{C}\right\}$ is not a vector space and the first possibility is ruled out. 
This means that $T$ must have the form given in (b). Let us take $f$ in $\mathcal{H}_{v_{0}}(U)$ and $\lambda \in \mathbb{C}$. Since $T$ is complex linear we have

$$
T_{1}(\lambda f) \otimes T_{2}(y)=T(\lambda f \otimes y)=\lambda T(f \otimes y)=\lambda T_{1}(f) \otimes T_{2}(y)
$$

for all $y \in E$. Thus $T_{1}$ is complex linear. A similar argument shows that $T_{2}$ is also complex linear. The result now follows from the first Banach-Stone theorem.

When $U$ is strictly convex it is possible to combine Theorem 3 and Theorem 8 to extend $\phi$ to all of $U$.

4. The isometry group. In Section 2 we proved that any isometry of $\mathcal{H}_{v_{0}}(U)$ is determined by a homeomorphism of $\mathcal{B}_{v}(U)$. Let $\operatorname{Aut}\left(\mathcal{B}_{v}(U)\right)$ denote the homeomorphisms of $\mathcal{B}_{v}(U)$.

REMARK 9. Let $U$ be a bounded open subset of $\mathbb{C}^{n}$ and $v$ be a continuous strictly positive weight which converges to 0 on the boundary of $U$. Then the set of $\phi \in \operatorname{Aut}\left(\mathcal{B}_{v}(U)\right)$ for which there is an isometry of $\mathcal{H}_{v_{0}}(U)$ of the form $T_{\phi}(f)(z)=h_{\phi}(z) f(\phi(z))$ is a subgroup of $\operatorname{Aut}\left(\mathcal{B}_{v}(U)\right)$.

Proof. Let $\phi, \psi$ belong to this set. Then

$$
T_{\psi}^{-1} \circ T_{\phi}(f)(z)=h_{\psi}\left(\psi^{-1}(z)\right)^{-1} h_{\phi}\left(\psi^{-1}(z)\right) f\left(\phi\left(\psi^{-1}(z)\right)\right)
$$

and the result is proved.

Definition 10. We denote by $\Lambda_{v}(U)$ the subgroup of $\phi$ in $\operatorname{Aut}\left(\mathcal{B}_{v}(U)\right)$ for which there is an isometry of $\mathcal{H}_{v_{0}}(U)$ of the form

$$
T_{\phi}(f)(z)=h_{\phi}(z) f(\phi(z)),
$$

and call it the isometry group of $v$.

Proposition 11. Let $U$ and $V$ be bounded open subsets of $\mathbb{C}^{n}$, and $v$ and $w$ be continuous strictly positive weights on $U$ and $V$ respectively which converge to 0 on the boundaries of their respective domains. If $\mathcal{H}_{v_{0}}(U)$ and $\mathcal{H}_{w_{0}}(V)$ are isometrically isomorphic then $\Lambda_{v}(U)$ is isomorphic to $\Lambda_{w}(V)$ as a topological group.

Proof. By our first Banach-Stone theorem (Theorem 1) we know that any isometry from $\mathcal{H}_{v_{0}}(U)$ onto $\mathcal{H}_{w_{0}}(V)$ has the form

$$
A_{\phi}(f)(z)=g_{\phi}(z) f(\phi(z)) \quad \text { for all } f \in \mathcal{H}_{v_{0}}(U), z \in \mathcal{B}_{w}(V),
$$

with some homeomorphism $\phi$ of $\mathcal{B}_{w}(V)$ onto $\mathcal{B}_{v}(U)$ and $g_{\phi} \in \mathcal{H}_{w_{0}}(V)$. Given $\psi \in \Lambda_{w}(V)$ we denote the associated isometry by $T_{\psi}$. Then $A_{\phi}^{-1} \circ T_{\psi} \circ A_{\phi}$ is an isometry of $\mathcal{H}_{v_{0}}(U)$ and

$$
\begin{aligned}
A_{\phi}^{-1} \circ T_{\psi} \circ A_{\phi}(f)(z) \\
\quad=g_{\phi}\left(\phi^{-1}(z)\right)^{-1} h_{\psi}\left(\phi^{-1}(z)\right) g_{\phi}\left(\psi \circ \phi^{-1}(z)\right) f\left(\phi \circ \psi \circ \phi^{-1}(z)\right)
\end{aligned}
$$


for all $f \in \mathcal{H}_{v_{0}}(U)$ and $z \in \mathcal{B}_{v}(U)$. Hence $\phi \circ \psi \circ \phi^{-1} \in \Lambda_{v}(U)$. As the mapping $\psi \mapsto \phi \circ \psi \circ \phi^{-1}$ is a group isomorphism and a homeomorphism, the result follows.

Let $\Omega$ be an open subset of $\mathbb{C}^{n}$ and $u \in \mathcal{C}^{2}(\Omega)$ be real-valued. We say that $u$ is pluriharmonic if

$$
\frac{\partial^{2} u}{\partial z_{j} \partial \bar{z}_{k}}=0
$$

on $\Omega$ for $j, k=1, \ldots, n$. If $f: \Omega \rightarrow \mathbb{C}$ is holomorphic then $u(z)=\log |f(z)|$ is pluriharmonic on $\Omega \backslash f^{-1}(0)$.

The condition in the following theorem should be compared with that in $\left[19\right.$, Theorem 1] for isometries on the weighted Bergman space $B_{F}^{p}(\Omega)$.

THEOREM 12. Let $v$ be a complete, continuous, strictly positive weight on a bounded simply connected open set $U$ which converges to 0 on the boundary of $U$. Then a homeomorphism $\phi \in \operatorname{Aut}(U)$ belongs to $\Lambda_{v}(U)$ if and only if $\log ((v \circ \phi) / v)$ is pluriharmonic.

Proof. First suppose that $\phi \in \Lambda_{v}(U)$. Then there is a holomorphic function $h_{\phi}: U \rightarrow \mathbb{C}$ such that

$$
T_{\phi}(f)(z):=h_{\phi}(z) f \circ \phi(z)
$$

is an isometry of $\mathcal{H}_{v_{0}}(U)$. Further, $\left|h_{\phi}\right|=(v \circ \phi) / v$ on $U$ and hence $\log ((v \circ \phi) / v)$ is pluriharmonic.

Conversely, suppose that $\log ((v \circ \phi) / v)$ is pluriharmonic. It follows from [30, Theorem 4.4.9] and the remark following it that there is a holomorphic function $g: U \rightarrow \mathbb{C}$ such that $v \circ \phi(z) / v(z)=\left|e^{g(z)}\right|$. Define $S: \mathcal{H}_{v_{0}}(U) \rightarrow$ $\mathcal{H}_{v_{0}}(U)$ by

$$
S(f)(z)=e^{g(z)} f \circ \phi(z) .
$$

Then

$$
\|S(f)\|_{v}=\sup _{z \in U} v(z)\left|e^{g(z)} f \circ \phi(z)\right|=\sup _{z \in U} v \circ \phi(z)|f \circ \phi(z)|=\|f\|_{v},
$$

and therefore $\phi \in \Lambda_{v}(U)$.

5. Determining isometries between spaces of weighted holomorphic functions on $\Delta$ with complete radial weights. For a twice differentiable function $u$ on an open subset of $\mathbb{C}$, the Laplacian $\Delta u$ of $u$ is defined by

$$
\triangle u=4 \frac{\partial^{2} u}{\partial z \partial \bar{z}} .
$$

In what follows we shall use the following two facts: 
1. If $k: \Delta \rightarrow \mathbb{C}$ is a twice differentiable radial function of $r$ then

$$
\triangle k=\frac{\partial^{2} k}{\partial r^{2}}+\frac{1}{r} \frac{\partial k}{\partial r}
$$

2. If $k: U \rightarrow \mathbb{C}$ is a twice differentiable function of $z$ and $\phi$ is holomorphic then

$$
\triangle(k \circ \phi)(a)=(\triangle k)(\phi(a))\left|\phi^{\prime}(a)\right|^{2} .
$$

(See the proof of [17, Corollary 2.5.7].)

Given $\alpha>0, \beta \geq 1$ we shall use $v_{\alpha, \beta}$ to denote the weight on $\Delta$ given by $\left(1-|z|^{\beta}\right)^{\alpha}$. In $\left[8\right.$, Examples 10] we proved that $v_{\alpha, \beta}$ is complete.

Theorem 13. Let $\alpha>0$ and $\beta \geq 1$.

(a) For $\beta \neq 2$, every isometry $T$ of $\mathcal{H}_{\left(v_{\alpha, \beta}\right)_{0}}(\Delta)$ and of $\mathcal{H}_{v_{\alpha, \beta}}(\Delta)$ has the form $T(f)(z)=\lambda f\left(e^{i \theta} z\right)$ for some $\lambda \in \Gamma$ and some $\theta \in \mathbb{R}$.

(b) Each isometry $T$ of $\mathcal{H}_{\left(v_{\alpha, 2}\right)_{0}}(\Delta)$ or $\mathcal{H}_{\left(v_{\alpha, 2}\right)}(\Delta)$ has the form $T(f)(z)$ $=\lambda \phi^{\prime}(z)^{\alpha} f(\phi(z))$ for some $\lambda \in \Gamma$ and some automorphism $\phi$ of $\Delta$.

Proof. (a) Suppose that $T$ is an isometry of $\mathcal{H}_{\left(v_{\alpha, \beta}\right)_{0}}(\Delta)$ or $\mathcal{H}_{v_{\alpha, \beta}}(\Delta)$. By Corollary 5 ,

$$
T(f)(z)=h_{\phi}(z) f(\phi(z))
$$

for all $f \in \mathcal{H}_{\left(v_{\alpha, \beta}\right)_{0}}(\Delta)$ or all $f \in \mathcal{H}_{v_{\alpha, \beta}}(\Delta)$, and all $z \in \Delta$. Then Theorem 12 tells us that

$$
\triangle \log v_{\alpha, \beta}(\phi(z))=\triangle \log v_{\alpha, \beta}(z)
$$

or that

$$
-\frac{\alpha \beta^{2}|\phi(z)|^{\beta-2}\left|\phi^{\prime}(z)\right|^{2}}{\left(1-|\phi(z)|^{\beta}\right)^{2}}=-\frac{\alpha \beta^{2}|z|^{\beta-2}}{\left(1-|z|^{\beta}\right)^{2}} .
$$

As the right-hand side is 0 when $z=0$, we see that $\phi(0)=0$ and hence $\phi$ is a rotation.

(b) It follows from Theorem 1 that there is an automorphism $\phi$ of $\Delta$ and an $h_{\phi}$ in $\mathcal{H}_{v_{0}}(\Delta)$ such that

$$
T_{\phi}(f)(z)=h_{\phi}(z) f \circ \phi(z)
$$

for all $z$ in $\Delta$. Theorem 12 tells us that $\phi \in \Lambda_{v_{\alpha, 2}}(\Delta)$ if and only if

$$
\triangle \log v_{\alpha, 2}(\phi(z))=\triangle \log v_{\alpha, 2}(z),
$$

which reduces to

$$
-\frac{4 \alpha\left|\phi^{\prime}(z)\right|^{2}}{\left(1-|\phi(z)|^{2}\right)^{2}}=-\frac{4 \alpha}{\left(1-|z|^{2}\right)^{2}} .
$$

Using the Schwarz-Pick theorem we see that this equation is satisfied by all $\phi$ in $\operatorname{Aut}(\Delta)$ and thus $\Lambda_{v_{\alpha, 2}}(\Delta)=\operatorname{Aut}(\Delta)$. The Schwarz-Pick theorem also gives

$$
\left|\phi^{\prime}(z)\right|^{2}=\frac{\left(1-|\phi(z)|^{2}\right)^{2}}{\left(1-|z|^{2}\right)^{2}}=\left|h_{\phi}(z)\right|^{2 / \alpha},
$$


where the final equality comes from Theorem 2. From the open mapping theorem we see that $h_{\phi}(z)=\lambda \phi^{\prime}(z)^{\alpha}$ for some $\lambda \in \Gamma$, and the result is proven.

Let us now determine when spaces of the form $\mathcal{H}_{\left(v_{\alpha, \beta}\right)_{0}}(\Delta)$ can be isometrically isomorphic.

Theorem 14. Let $\alpha, \alpha^{\prime}>0$ and $\beta, \beta^{\prime} \geq 1$. Then $\mathcal{H}_{\left(v_{\alpha, \beta}\right)_{0}}(\Delta)$ is isometrically isomorphic to $\mathcal{H}_{\left(v_{\alpha^{\prime}, \beta^{\prime}}\right)_{0}}(\Delta)$ if and only if $\alpha=\alpha^{\prime}$ and $\beta=\beta^{\prime}$.

Proof. Suppose that $T$ is an isometry from $\mathcal{H}_{\left(v_{\alpha, \beta}\right)_{0}}(\Delta)$ onto $\mathcal{H}_{\left(v_{\alpha^{\prime}, \beta^{\prime}}\right)_{0}}(\Delta)$. By the first Banach-Stone theorem,

$$
T(f)(z)=h_{\phi}(z) f(\phi(z)) \quad \text { for all } f \in \mathcal{H}_{\left(v_{\alpha, \beta}\right)_{0}}(\Delta), z \in \Delta,
$$

where $h_{\phi} \in \mathcal{H}_{\left(v_{\alpha^{\prime}, \beta^{\prime}}\right)_{0}}(\Delta)$ and $\phi$ is an automorphism of $\Delta$. Furthermore,

$$
\left|h_{\phi}(z)\right|=\frac{v_{\alpha, \beta}(\phi(z))}{v_{\alpha^{\prime}, \beta^{\prime}}(z)} .
$$

By the argument given in Theorem 13 we see that when $\beta, \beta^{\prime} \neq 2$ we obtain

$$
-\frac{\alpha \beta^{2}|\phi(z)|^{\beta-2}\left|\phi^{\prime}(z)\right|^{2}}{\left(1-|\phi(z)|^{\beta}\right)^{2}}=-\frac{\alpha^{\prime}\left(\beta^{\prime}\right)^{2}|z|^{\beta^{\prime}-2}}{\left(1-|z|^{\beta^{\prime}}\right)^{2}} .
$$

As the right-hand side is 0 when $z=0$ we see that $\phi(0)=0$ and hence $\phi$ is a rotation. Let $z=r e^{i \theta}$. Then $|\phi(z)|=r$ and putting this into $(*)$ gives

$$
\alpha \beta^{2} \frac{r^{\beta-2}}{\left(1-r^{\beta}\right)^{2}}=\alpha^{\prime}\left(\beta^{\prime}\right)^{2} \frac{r^{\beta^{\prime}-2}}{\left(1-r^{\beta^{\prime}}\right)^{2}}
$$

or in other words

$$
\alpha \beta^{2}\left(r^{\beta-2}-2 r^{\beta+\beta^{\prime}-2}+r^{2 \beta^{\prime}+\beta-2}\right)=\alpha^{\prime}\left(\beta^{\prime}\right)^{2}\left(r^{\beta^{\prime}-2}-2 r^{\beta^{\prime}+\beta-2}+r^{2 \beta+\beta^{\prime}-2}\right)
$$

for all $r \in(0,1)$. Equating leading powers of $r$ we see that $\beta=\beta^{\prime}$. Now equate coefficient of powers of $r$ to conclude that $\alpha=\alpha^{\prime}$.

Next suppose that $\beta=2 \neq \beta^{\prime}$. As $\Lambda_{v_{\alpha, 2}}(\Delta)$ is $\operatorname{Aut}(\Delta)$, and $\Lambda_{v_{\alpha^{\prime}, \beta^{\prime}}}(\Delta)$ is the group of rotations of $\Delta$, Proposition 11 implies that $\mathcal{H}_{\left(v_{\alpha, \beta}\right)_{0}}(\Delta)$ is not isometrically isomorphic to $\mathcal{H}_{\left(v_{\alpha, \beta^{\prime}}\right)_{0}}(\Delta)$.

Finally, suppose that $\beta=\beta^{\prime}=2$ and $\mathcal{H}_{\left(v_{\alpha, 2}\right)_{0}}(\Delta)$ is isometrically isomorphic to $\mathcal{H}_{v_{\left(\alpha^{\prime}, 2\right)_{0}}}(\Delta)$ via an isometry $T_{\phi}$. Then

$$
\triangle\left(\log \left(1-|\phi(z)|^{2}\right)^{\alpha}\right)=\triangle\left(\log \left(1-|z|^{2}\right)^{\alpha^{\prime}}\right)
$$

for all $z \in \Delta$. By using the chain rule this gives

$$
\frac{\alpha\left|\phi^{\prime}(z)\right|^{2}}{\left(1-|\phi(z)|^{2}\right)^{2}}=\frac{\alpha^{\prime}}{\left(1-|z|^{2}\right)^{2}}
$$

Applying the Schwarz-Pick theorem we deduce that $\alpha=\alpha^{\prime}$, and the proof is complete. 
This example also shows that the converse to Proposition 11 is false. Consider $v_{1, \beta}=1-|z|^{\beta}$ and $v_{1, \beta^{\prime}}=1-|z|^{\beta^{\prime}}$ where $\beta \neq \beta^{\prime}$ and both are different from 2 . As we have just seen, $\Lambda_{v_{1, \beta}}(\Delta)=\Lambda_{v_{1, \beta^{\prime}}}(\Delta)$ is the group of all rotations about the origin. However, $\mathcal{H}_{\left(v_{1, \beta}\right)_{0}}(\Delta)$ and $\mathcal{H}_{\left(v_{1, \beta^{\prime}}\right)_{0}}(\Delta)$ are not isometrically isomorphic.

Theorem 15. For $\beta \geq 1$, let $w_{\beta}(z)$ denote the radial weight on the unit disc given by $w_{\beta}(z)=e^{-1 /\left(1-|z|^{\beta}\right)}$. Then

(a) for $\beta \neq \beta^{\prime}$ there is no isometry from $\mathcal{H}_{\left(w_{\beta}\right)_{0}}(\Delta)$ onto $\mathcal{H}_{\left(w_{\beta^{\prime}}\right)_{0}}(\Delta)$,

(b) every isometry $T$ from $\mathcal{H}_{\left(w_{\beta}\right)_{0}}(\Delta)$ onto $\mathcal{H}_{\left(w_{\beta}\right)_{0}}(\Delta)$ and of $\mathcal{H}_{w_{\beta}}(\Delta)$ onto $\mathcal{H}_{w_{\beta}}(\Delta)$ has the form $T(f)(z)=\lambda f\left(e^{i \theta} z\right)$ for some $\lambda \in \mathbb{C}$ with $|\lambda|=1$ and some $\theta \in \mathbb{R}$.

Proof. Suppose that $T$ is an isometry from $\mathcal{H}_{\left(w_{\beta}\right)_{0}}(\Delta)$ onto $\mathcal{H}_{\left(w_{\beta^{\prime}}\right)_{0}}(\Delta)$. By the first Banach-Stone theorem,

$$
T(f)(z)=h_{\phi}(z) f(\phi(z)) \quad \text { for all } f \in \mathcal{H}_{\left(w_{\beta}\right)_{0}}(\Delta), z \in \Delta,
$$

where $h_{\phi} \in \mathcal{H}_{\left(w_{\beta^{\prime}}\right)_{0}}(\Delta)$. Furthermore,

$$
\left|h_{\phi}(z)\right|=\frac{w_{\beta}(\phi(z))}{w_{\beta^{\prime}}(z)} .
$$

By the same argument as used in Theorem 13 we find that when $\beta, \beta^{\prime}>2$,

$$
\beta^{2} \frac{\left(|\phi(z)|^{2(\beta-1)}+|\phi(z)|^{\beta-2}\right)\left|\phi^{\prime}(z)\right|^{2}}{\left(1-|\phi(z)|^{\beta}\right)^{3}}=\left(\beta^{\prime}\right)^{2} \frac{|z|^{2\left(\beta^{\prime}-1\right)}+|z|^{\beta^{\prime}-2}}{\left(1-|z|^{\beta^{\prime}}\right)^{3}} .
$$

As the right-hand side is 0 when $z=0$, we see that $\phi(0)=0$ and hence $\phi(z)$ is a rotation. In particular, $|\phi(z)|=|z|$. Letting $z=r e^{i \theta}$ we get

$$
\left(1-r^{\beta}\right)^{3}\left(\beta^{\prime}\right)^{2}\left(r^{2 \beta^{\prime}}+r^{\beta^{\prime}}\right)=\left(1-r^{\beta^{\prime}}\right)^{3} \beta^{2}\left(r^{2 \beta}+r^{\beta}\right) .
$$

Equating lowest powers on both sides we see that $\beta=\beta^{\prime}$. An analogous argument works for $\beta, \beta^{\prime}<2$ and $\beta<2<\beta^{\prime}$.

Next suppose that $\beta>\beta^{\prime}=2$. In this case the equation

$$
\triangle \log w_{\beta}(\phi(z))=\triangle \log w_{\beta^{\prime}}(z)
$$

gives

$$
\beta^{2} \frac{\left(|\phi(z)|^{2(\beta-1)}+|\phi(z)|^{\beta-2}\right)\left|\phi^{\prime}(z)\right|^{2}}{\left(1-|\phi(z)|^{\beta}\right)^{3}}=4 \frac{|z|^{2}+1}{\left(1-|z|^{2}\right)^{3}} .
$$

As there is $z_{0} \in \Delta$ such that $\phi\left(z_{0}\right)=0$ but the right-hand side is never 0 we see that the above equation cannot be solved and hence there is no isometry from $\mathcal{H}_{\left(w_{\beta}\right)_{0}}(\Delta)$ onto $\mathcal{H}_{\left(w_{2}\right)_{0}}(\Delta), \beta \neq 2$.

Finally, suppose that $\beta=\beta^{\prime}=2$. In this case the equation

$$
\triangle \log w_{\beta}(\phi(z))=\triangle \log w_{\beta^{\prime}}(z)
$$


implies that

$$
4 \frac{\left(|\phi(z)|^{2}+1\right)\left|\phi^{\prime}(z)\right|^{2}}{\left(1-|\phi(z)|^{2}\right)^{3}}=4 \frac{|z|^{2}+1}{\left(1-|z|^{2}\right)^{3}} .
$$

This can be rewritten, using the Schwarz-Pick theorem, as

$$
\frac{1-|\phi(z)|^{2}}{1-|z|^{2}}=\frac{1+|\phi(z)|^{2}}{1+|z|^{2}} \text {. }
$$

Solving this we get $|\phi(z)|=|z|$ for all $z \in \Delta$, and hence $\phi$ is a rotation.

THEOREM 16. Let $v(z)$ denote either of the radial weights on $\Delta$ given by $v_{1}(z)=(1-\log (1-|z|))^{\beta}, \beta<0$, and $v_{2}(z)=\arccos (|z|)$. Every isometry from $\mathcal{H}_{v_{0}}(\Delta)$ onto $\mathcal{H}_{v_{0}}(\Delta)$ and from $\mathcal{H}_{v}(\Delta)$ onto $\mathcal{H}_{v}(\Delta)$ is induced by a rotation of $\Delta$.

Proof. This follows as above from the fact that

$$
\begin{aligned}
\triangle \log \left(v_{1}(|z|)\right) & =\beta\left(\frac{1}{(1-|z|)^{2}(1-\log (1-|z|))}\right. \\
& \left.-\frac{1}{(1-|z|)^{2}(1-\log (1-|z|))^{2}}+\frac{1}{|z|(1-|z|)(1-\log (1-|z|))}\right)
\end{aligned}
$$

while

$$
\triangle \log \left(v_{2}(|z|)\right)=\frac{\sqrt{1-|z|^{2}}|z|+\arccos (|z|)}{|z| \arccos (|z|)^{2} \sqrt{1-|z|^{2}}\left(1-|z|^{2}\right)} .
$$

Isometry groups for weights in higher dimensions are calculated in [9].

6. Weights with isometry group $\operatorname{Aut}(\Delta)$. We have seen that the continuous strictly positive complete radial weight $v_{\alpha, 2}(z)=\left(1-|z|^{2}\right)^{\alpha}$ on the unit disc $\Delta$ has the property that its isometry group $\Lambda_{v}(\Delta)$ is equal to $\operatorname{Aut}(\Delta)$. In this section we examine the converse of this result.

We recall that if $U$ is a bounded open subset of $\mathbb{C}^{n}$ and $v$ is a continuous strictly positive weight which converges to 0 on the boundary of $U$, then $z$ in $U$ is said to be a $v$-peak point if there is $f$ in the unit ball of $\mathcal{H}_{v_{0}}(U)$ with $v(z) f(z)=1$ and $v(w)|f(w)|<1$ for all $w$ in $U \backslash\{z\}$. In [8, Theorem 3] we prove that $z$ is a $v$-peak point if and only if $v(z) \delta_{z}$ is a weak ${ }^{*}$-exposed point of $B_{\mathcal{H}_{v_{0}}(U)^{\prime}}$. Furthermore, by [8, Theorem 3], the set of weak*-strongly exposed points and the set of weak ${ }^{*}$-exposed points of $B_{\mathcal{H}_{v_{0}}(U)^{\prime}}$ coincide.

THEOREM 17. Let $v$ be a continuous strictly positive twice differentiable weight on $\Delta$ which converges to 0 on the boundary of $\Delta$. Then $\Lambda_{v}(\Delta)=$ $\operatorname{Aut}(\Delta)$ if and only if $v(z)=e^{k(z)}\left(1-|z|^{2}\right)^{\alpha}$ for some bounded real-valued harmonic function $k$ on $\Delta$ and some $\alpha>0$.

Proof. Suppose that $v(z)=e^{k(z)}\left(1-|z|^{2}\right)^{\alpha}$ for some bounded real-valued harmonic function $k$ on $\Delta$ and some $\alpha>0$. Let $g$ be a bounded analytic 
function with real part $k$. For $\phi \in \operatorname{Aut}(\Delta)$ and $f \in \mathcal{H}_{v_{0}}(\Delta)$ define $T_{\phi}(f)$ by

$$
T_{\phi}(f)(z)=\phi^{\prime}(z)^{\alpha} e^{g \circ \phi(z)-g(z)} f \circ \phi(z) .
$$

Then

$$
\begin{aligned}
\left\|T_{\phi}(f)\right\|_{v} & =\sup _{z \in \Delta} v(z)\left|\phi^{\prime}(z)^{\alpha} e^{g \circ \phi(z)-g(z)} f \circ \phi(z)\right| \\
& =\sup _{z \in \Delta}\left(1-|\phi(z)|^{2}\right)^{\alpha} e^{k \circ \phi(z)}|f \circ \phi(z)|=\|f\|_{v},
\end{aligned}
$$

and therefore $T_{\phi}$ is an isometry of $\mathcal{H}_{v_{0}}(\Delta)$.

Conversely, suppose that $\Lambda_{v}(\Delta)=\operatorname{Aut}(\Delta)$. We first observe that since $z$ in $U$ is a $v$-peak point if and only if $v(z) \delta_{z}$ is a weak ${ }^{*}$-exposed point of the unit ball of $\mathcal{H}_{v_{0}}(U)^{\prime}$, the set of $v$-peak points is non-empty. Therefore we can find $z_{0} \in \Delta$ and $f \in \mathcal{H}_{v_{0}}(\Delta)$ so that $v\left(z_{0}\right) f\left(z_{0}\right)=1$ and $v(z)|f(z)|<1$ for all $z \in \Delta \backslash\left\{z_{0}\right\}$. Given $w_{0} \in \Delta$ we can find an automorphism $\phi$ of $\Delta$ so that $w_{0}=\phi\left(z_{0}\right)$. Let $g(z)=\lambda h_{\phi^{-1}}(z) f \circ \phi^{-1}(z)$ where $\lambda$ is a complex number of modulus 1 chosen so that $g\left(w_{0}\right)$ is real. Then

$$
v\left(w_{0}\right) g\left(w_{0}\right)=v\left(w_{0}\right) \frac{v\left(\phi^{-1}\left(w_{0}\right)\right)}{v\left(w_{0}\right)} f \circ \phi^{-1}\left(w_{0}\right)=v\left(z_{0}\right) f\left(z_{0}\right)=1
$$

and

$$
v(w)|g(w)|=v(w) \frac{v\left(\phi^{-1}(w)\right)}{v(w)}\left|f \circ \phi^{-1}(w)\right|=v(z)|f(z)|<1
$$

for $w \in \Delta \backslash\left\{w_{0}\right\}$. Therefore each point of $\Delta$ is a $v$-peak point and so $v$ is complete.

We now observe that for any $\phi \in \operatorname{Aut}(\Delta)$ and $z \in \Delta$ we have

$$
\triangle(\log \circ v \circ \phi)(z)=\triangle(\log \circ v)(z) .
$$

From the "chain rule" for Laplacians we get

$$
\frac{\triangle(\log \circ v)(\phi(z))}{\triangle(\log \circ v)(z)}=\frac{1}{\left|\phi^{\prime}(z)\right|^{2}}
$$

for all $\phi \in \operatorname{Aut}(\Delta)$. But taking the classical weight $v_{1,2}(z)=1-|z|^{2}$, we also find that

$$
\frac{\triangle\left(\log \circ v_{1,2}\right)(\phi(z))}{\triangle\left(\log \circ v_{1,2}\right)(z)}=\frac{1}{\left|\phi^{\prime}(z)\right|^{2}}
$$

for all $\phi \in \operatorname{Aut}(\Delta)$ and $z \in \Delta$. Hence

$$
\frac{\triangle(\log \circ v)(\phi(z))}{\triangle\left(\log \circ v_{1,2}\right)(\phi(z))}=\frac{\triangle(\log \circ v)(z)}{\triangle\left(\log \circ v_{1,2}\right)(z)}
$$

for all $\phi \in \operatorname{Aut}(\Delta)$ and $z \in \Delta$. Since $\operatorname{Aut}(\Delta)$ acts transitively on $\Delta$ we get

$$
\frac{\triangle(\log \circ v)(z)}{\triangle\left(\log \circ v_{1,2}\right)(z)}=\alpha
$$


for some $\alpha \in \mathbb{R}$ and all $z \in \Delta$. As $\log \circ v_{1,2}$ is superharmonic and $v$ tends to 0 as $z$ tends to the boundary of $\Delta$, it follows that $(1 / \alpha) \log \circ v$ is also superharmonic. Applying Weyl's lemma ([28, Lemma 3.7]) we deduce that there is a harmonic function $k: \Delta \rightarrow \mathbb{R}$ so that

$$
\log v(z)=\log \left(v_{1,2}(z)^{\alpha}\right)+k(z)
$$

for all $z$ in $\Delta$. Hence

$$
v(z)=e^{k(z)}\left(1-|z|^{2}\right)^{\alpha}
$$

for all $z \in \Delta$, some bounded real-valued harmonic function $k$ on $\Delta$ and some $\alpha \in \mathbb{R}$. The boundary condition on $v$ implies that $\alpha>0$.

COROLlary 18. Let $v$ be a continuous strictly positive twice differentiable radial weight on $\Delta$ which converges to 0 on the boundary of $\Delta$. If $\Lambda_{v}(\Delta)=\operatorname{Aut}(\Delta)$ then $v(z)=\lambda\left(1-|z|^{2}\right)^{\alpha}$ for some $\lambda \in \mathbb{R}^{+}$and some $\alpha>0$.

Proof. The result follows from Theorem 17 and the fact that the only radial harmonic functions are the constant functions.

7. The isometry cocycle. We note that given $\phi \in \Lambda_{v}(U)$ the function $h_{\phi}$ with the property that there is an isometry of $\mathcal{H}_{v_{0}}(U)$ of the form

$$
T_{\phi}(f)(z)=h_{\phi}(z) f(\phi(z))
$$

is not unique. Indeed, $\lambda h_{\phi}$ will also give an isometry of $\mathcal{H}_{v_{0}}(U)$ for any $\lambda \in \Gamma$. However, since

$$
\left|h_{\phi}(z)\right|=\frac{v \circ \phi(z)}{v(z)}
$$

$\left|h_{\phi}(z)\right|$ is uniquely determined by $\phi$.

Given groups $G_{1}$ and $G_{2}$ with identities $e_{1}$ and $e_{2}$ respectively and an action of $G_{1}$ on a set $S$, a cocycle $\pi$ from $G_{1} \times S$ to $G_{2}$ is a function $\pi: G_{1} \times S \rightarrow G_{2}$ such that

(a) $\pi\left(e_{1}, s\right)=e_{2}$ for all $s$ in $S$,

(b) $\pi(g h, s)=\pi(g, h s) \pi(h, s)$ for all $g, h$ in $G_{1}$ and all $s$ in $S$.

Cocycles $\pi_{1}: G_{1} \times S \rightarrow G_{2}$ and $\pi_{2}: G_{1} \times S \rightarrow G_{2}$ are said to be cohomologous if there is $a: S \rightarrow G_{2}$ so that

$$
\pi_{2}(g, s)=a(s) \pi_{1}(g, s) a(g s)^{-1} \quad \text { for all } g \in G_{1}, s \in S .
$$

Proposition 19. Let $U$ be a bounded open subset of $\mathbb{C}^{n}$ and $v$ be a continuous strictly positive weight which converges to 0 on the boundary of $U$. The mapping $\pi_{v}: \Lambda_{v}(U) \times \mathcal{B}_{v}(U) \rightarrow\left(\mathbb{R}^{+}, \times\right), \pi_{v}(\phi, z)=\left|h_{\phi}(z)\right|$, is a cocycle. 
Proof. Let $\phi, \psi$ belong to $\Lambda_{v}(U)$. By Theorem $1, T_{\phi} \circ T_{\psi}=T_{\eta}$ for some $\eta$ in $\Lambda_{v}(U)$. For all $f \in \mathcal{H}_{v_{0}}(U)$ and all $z \in \mathcal{B}_{v}(U)$ we have

$$
h_{\psi}(z) h_{\phi}(\psi(z)) f(\phi \circ \psi(z))=h_{\eta}(z) f(\eta(z)) .
$$

Taking $f \equiv 1$ we get

$$
h_{\psi}(z) h_{\phi}(\psi(z))=h_{\eta}(z) .
$$

Now taking $f(z)=z_{j}$ for $j=1, \ldots, n$, using $(*)$ and $(* *)$, we get

$$
\eta=\phi \circ \psi \text {. }
$$

Putting this into $(* *)$ we obtain

$$
\left|h_{\phi \circ \psi}(z)\right|=\left|h_{\psi}(z)\right|\left|h_{\phi}(\psi(z))\right|
$$

or equivalently

$$
\pi_{v}(\phi \circ \psi, z)=\pi_{v}(\psi, z) \pi_{v}(\phi, \psi(z)) .
$$

DeFinition 20. We shall call $\pi_{v}$ the isometry cocycle of $v$.

EXAMPLE 21.

(a) For $v_{\alpha, \beta}(z)=\left(1-|z|^{\beta}\right)^{\alpha}, \alpha>0, \beta \geq 1, \beta \neq 2$ and $w(z)=e^{-1 /\left(1-|z|^{\beta}\right)}$ we have seen that $\Lambda_{v}(\Delta)$ is the group of all rotations about the origin and that $\pi_{v}(\phi, z)=1$ for all $\phi \in \Lambda_{v}(U)$ and all $z \in \Delta$.

(b) We have seen that $\Lambda_{v_{\alpha, 2}}(\Delta)=\operatorname{Aut}(\Delta)$ and $\pi_{v_{\alpha, 2}}(\phi, z)=\left|\phi^{\prime}(z)\right|^{\alpha}$.

Proposition 22. Let $U$ and $V$ be bounded open subsets of $\mathbb{C}^{n}$, and $v$ and $w$ be continuous strictly positive weights on $U$ and $V$ respectively which converge to 0 on their respective boundaries. If $A_{\phi}$ is an isometric isomorphism from $\mathcal{H}_{v_{0}}(U)$ onto $\mathcal{H}_{w_{0}}(V), A_{\phi}(f)(z)=g_{\phi}(z) f(\phi(z))$, then the cocycles $\pi_{v}(\psi, z)$ and $\pi_{w}\left(\phi^{-1} \circ \psi \circ \phi, \phi^{-1}(z)\right)$ on $\Lambda_{v}(U) \times \mathcal{B}_{v}(U)$ are cohomologous.

Proof. Let $\psi \in \Lambda_{v}(U)$. Then $A_{\phi} \circ T_{\psi} \circ A_{\phi}^{-1}(f)$ is an isometry of $\mathcal{H}_{w_{0}}(V)$ and it follows as in the proof of Proposition 11 that

$$
A_{\phi} \circ T_{\psi} \circ A_{\phi}^{-1}(f)(w)=g_{\phi}(w) h_{\psi}(\phi(w)) g_{\phi^{-1}}(\psi \circ \phi(w)) f\left(\phi^{-1} \circ \psi \circ \phi(w)\right)
$$

for all $f \in \mathcal{H}_{w_{0}}(V)$ and $w \in \mathcal{B}_{w}(V)$. Since the cocycle of $A_{\phi} \circ T_{\psi} \circ A_{\phi}^{-1}$ is $\pi_{w}\left(\phi^{-1} \circ \psi \circ \phi, w\right)$ we see that

$$
\pi_{w}\left(\phi^{-1} \circ \psi \circ \phi, \phi^{-1}(z)\right)=g_{\phi}\left(\phi^{-1}(z)\right) \pi_{v}(\psi, z) g_{\phi}\left(\phi^{-1} \circ \psi(z)\right)^{-1} .
$$

Thus the cocycles are cohomologous.

The converse of Proposition 22 is false. To see this, let $\beta \geq 1$ and $\alpha, \alpha^{\prime}>0$, $\alpha \neq \alpha^{\prime}$. Consider the weights $v_{\alpha, \beta}$ and $v_{\alpha^{\prime}, \beta}$; we claim that $\pi_{v_{\alpha, \beta}}$ and $\pi_{v_{\alpha^{\prime}, \beta}}$ are cohomologous. Indeed, when $\beta \neq 2$ we notice that $\pi_{v_{\alpha, \beta}}(\phi, z)=\pi_{v_{\alpha^{\prime}, \beta}}(\phi, z)$ $\equiv 1$ and hence are clearly cohomologous. When $\beta=2$ we have

$$
\pi_{v_{\alpha, 2}}(\phi, z)=\frac{\left(1-|\phi(z)|^{2}\right)^{\alpha}}{\left(1-|z|^{2}\right)^{\alpha}} \quad \text { and } \quad \pi_{v_{\alpha^{\prime}, 2}}(\phi, z)=\frac{\left(1-|\phi(z)|^{2}\right)^{\alpha^{\prime}}}{\left(1-|z|^{2}\right)^{\alpha^{\prime}}} .
$$


Taking $a(z)=\left(1-|z|^{2}\right)^{\alpha^{\prime}-\alpha}$ gives us a cohomology between $\pi_{v_{\alpha, 2}}$ and $\pi_{v_{\alpha^{\prime}, 2}}$. We summarise our invariants in the following table:

\begin{tabular}{ccc}
\hline \multicolumn{3}{c}{ Isometry invariants } \\
\hline Invariant & Symbol & Tolerance \\
\hline$v$-boundary & $\mathcal{B}_{v}(U)$ & homeomorphism \\
interior of $v$-boundary & $\check{\mathcal{B}}_{v}(U)$ & conformality \\
isometry group & $\Lambda_{v}(U)$ & topological isomorphism \\
isometry cocycle & $\pi_{v}$ & cohomology \\
\hline
\end{tabular}

8. Weights whose isometry groups contain all rotations. Let $U$ be an open subset of $\mathbb{C}^{n}$ and $v$ be a continuous strictly positive weight which converges to 0 on the boundary of $U$. We define $w: U \rightarrow \mathbb{R}$ by $w(z)=1 / v(z)$. The closed unit ball of $\mathcal{H}_{v_{0}}(U)$ is $\left\{f \in \mathcal{H}_{v_{0}}(U):|f(z)| \leq\right.$ $w(z)$ for all $z \in U\}$ whereas the closed unit ball of $\mathcal{H}_{v}(U)$ is $\left\{f \in \mathcal{H}_{v}(U)\right.$ : $|f(z)| \leq w(z)$ for all $z \in U\}$. We define $\widetilde{w}_{0}: U \rightarrow \mathbb{R}$ by

$$
\widetilde{w}_{0}(z)=\sup \left\{|f(z)|: f \in B_{\mathcal{H}_{v_{0}}(U)}\right\}
$$

and $\widetilde{w}: U \rightarrow \mathbb{R}$ by

$$
\widetilde{w}(z)=\sup \left\{|f(z)|: f \in B_{\mathcal{H}_{v}(U)}\right\} .
$$

Let $\widetilde{v}_{0}(z)=1 / \widetilde{w}_{0}(z)$ and $\widetilde{v}(z)=1 / \widetilde{w}(z)$. Then $\widetilde{v}_{0}$ and $\widetilde{v}$ are continuous strictly positive weights which satisfy $0<v \leq \widetilde{v} \leq \widetilde{v}_{0}$. Moreover, when $U=\Delta$ it follows from [5, Proposition 1.1] that $\widetilde{v}$ also converges to 0 on the boundary of $\Delta$. The spaces $\mathcal{H}_{v}(U)$ and $\mathcal{H}_{\widetilde{v}}(U)$ are isometrically isomorphic, as are $\mathcal{H}_{v_{0}}(U)$ and $\mathcal{H}_{\widetilde{v}_{0}}(U)$. We note that $\widetilde{v}$ and $\widetilde{v}_{0}$ are radial whenever $U$ is balanced and $v$ is radial. Furthermore, Hadamard's three circles theorem implies that $\log \widetilde{w}_{0}$ and $\log \widetilde{w}$ are convex functions of $\log |z|$. When $v$ is a continuous decreasing radial weight on the unit disc, [28, Theorem 2.6.6] tells us that $\log \widetilde{w}_{0}$ and $\log \widetilde{w}$ are subharmonic. $e^{i \theta} z$.

Given a balanced domain $U$ and $\theta$ in $\mathbb{R}$ we define $R_{\theta}: U \rightarrow U$ by $R_{\theta}(z)=$

TheOREM 23. Let $v: \Delta \rightarrow \mathbb{R}$ be a continuous strictly positive weight which converges to 0 on the boundary of $\Delta$ and such that $\mathcal{B}_{v}(\Delta)$ has an accumulation point in $\Delta$. For $\theta \in \mathbb{R}$ the following are equivalent:

(a) $\triangle \log \widetilde{v}_{0} \circ R_{\theta}=\triangle \log \widetilde{v}_{0}$,

(b) $R_{\theta} \in \Lambda_{v}(\Delta)$,

(c) $R_{\theta} \in \Lambda_{\widetilde{v}_{0}}(\Delta)$.

Proof. Since $\mathcal{H}_{v_{0}}(\Delta)$ is isometrically isomorphic to $\mathcal{H}_{\widetilde{v}_{0}}(\Delta)$ we see that (b) and (c) are equivalent. Suppose that (b) holds. It follows from Theorem 1 that there is $h_{\theta} \in \mathcal{H}_{v_{0}}(\Delta)$ such that there is an isometry, $T_{\theta}$, of $\mathcal{H}_{v_{0}}(\Delta)$ of 
the form

$$
T_{\theta}(f)(z)=h_{\theta}(z) f \circ R_{\theta}(z) \quad \text { for all } f \in \mathcal{H}_{v_{0}}(\Delta), z \in \Delta .
$$

For $z \in \Delta$ we have

$$
\left\langle f, T_{\theta}^{*} \delta_{z}\right\rangle=\left\langle T_{\theta} f, \delta_{z}\right\rangle=\left\langle h_{\theta} f \circ R_{\theta}, \delta_{z}\right\rangle=\left\langle f, h_{\theta}(z) \delta_{R_{\theta}(z)}\right\rangle
$$

for all $f \in \mathcal{H}_{v_{0}}(\Delta)$. Since $T_{\theta}^{*}$ is an isometry we notice that

$$
1 / \widetilde{v}_{0}(z)=\left\|\delta_{z}\right\|=\left|h_{\theta}(z)\right|\left\|\delta_{R_{\theta}(z)}\right\|=\left|h_{\theta}(z)\right| \cdot 1 / \widetilde{v}_{0}\left(R_{\theta}(z)\right)
$$

for all $z \in \Delta$. Thus we see that

$$
\left|h_{\theta}(z)\right|=\frac{\widetilde{v}_{0}\left(R_{\theta}(z)\right)}{\widetilde{v}_{0}(z)} \quad \text { for all } z \in \Delta .
$$

Applying the Laplacian operator to the superharmonic functions $\log \widetilde{v}_{0} \circ R_{\theta}$ and $\log \widetilde{v}_{0}$ we get $\triangle \log \widetilde{v}_{0} \circ R_{\theta}=\triangle \log \widetilde{v}_{0}$.

Now suppose that (a) is true. Since $\log \widetilde{v}_{0}$ is superharmonic we can apply Weyl's lemma ([28, Lemma 3.7]) to conclude that there is a harmonic function $k_{\theta}: \Delta \rightarrow \mathbb{C}$ so that

$$
\log \widetilde{v}_{0}\left(R_{\theta}(z)\right)=\log \widetilde{v}_{0}(z)+k_{\theta}(z) \quad \text { for all } z \in \Delta .
$$

Then $\widetilde{v}_{0}\left(R_{\theta}(z)\right)=e^{k_{\theta}(z)} \widetilde{v}_{0}(z)$. Let $g_{\theta}: \Delta \rightarrow \mathbb{C}$ be a holomorphic function such that $k_{\theta}=\operatorname{Re} g_{\theta}$. Define $T_{\theta}: \mathcal{H}_{v_{0}}(\Delta) \rightarrow \mathcal{H}_{v_{0}}(\Delta)$ by

$$
T_{\theta}(f)(z)=e^{g_{\theta}(z)} f \circ R_{\theta}(z) \quad \text { for } z \in \Delta \text {. }
$$

Then

$$
\begin{aligned}
\left\|T_{\theta}(f)\right\|_{v} & =\sup _{z \in \Delta} v(z)\left|e^{g_{\theta}(z)} f \circ R_{\theta}(z)\right|=\sup _{z \in \Delta} \widetilde{v}_{0}(z) e^{k_{\theta}(z)}\left|f \circ R_{\theta}(z)\right| \\
& =\sup _{z \in \Delta} \widetilde{v}_{0} \circ R_{\theta}(z)\left|f \circ R_{\theta}(z)\right|=\|f\|_{v},
\end{aligned}
$$

proving that $T_{\theta}$ is an isometry of $\mathcal{H}_{v_{0}}(\Delta)$ onto $\mathcal{H}_{v_{0}}(\Delta)$ and therefore $R_{\theta} \in$ $\Lambda_{v}(\Delta)$.

Corollary 24. Let $v: \Delta \rightarrow \mathbb{R}$ be a continuous strictly positive weight which converges to 0 on the boundary of $\Delta$ and such that $\mathcal{B}_{v}(\Delta)$ has an accumulation point in $\Delta$. Then $\left\{R_{\theta}: \theta \in[0,2 \pi]\right\} \subset \Lambda_{v}(\Delta)$ if and only if $\triangle \log \circ \widetilde{v}_{0}$ is radial.

In practice, however, it is difficult to calculate $\widetilde{v}_{0}$ when given an arbitrary weight $v$. We therefore state the following corollary whose proof is based on Theorem 23.

Corollary 25. Let $v$ be a continuous strictly positive weight on $\Delta$ which converges to 0 on the boundary of $\Delta$. If $R_{\theta} \in \Lambda_{v}(\Delta)$ then $\triangle \log v\left(e^{i \theta} z\right)$ $=\triangle \log v(z)$ for all $z \in \mathcal{B}_{v}(\Delta)$. Moreover, if $v$ is complete then the converse holds. In particular, when $v$ is complete, $\left\{R_{\theta}: \theta \in[0,2 \pi]\right\} \subseteq \Lambda_{v}(\Delta)$ if and only if $\triangle \log \circ v$ is radial. 
We note the following lemma which is proved in an analogous way to part of Theorem 23.

Lemma 26. Let $v, w: \Delta \rightarrow \mathbb{R}$ be continuous strictly positive weights which converge to 0 on the boundary of $\Delta$ and such that $\mathcal{B}_{v}(\Delta)$ and $\mathcal{B}_{w}(\Delta)$ have each an accumulation point in $\Delta$. If $T(f)(z)=h_{\phi}(z) f(\phi(z))$ defines an isometric isomorphism between $\mathcal{H}_{v_{0}}(\Delta)$ and $\mathcal{H}_{w_{0}}(\Delta)$ then

$$
\left|h_{\phi}(z)\right|=\frac{\widetilde{w}_{0}(\phi(z))}{\widetilde{v}_{0}(z)} \quad \text { for all } z \in \Delta .
$$

\section{Determining isometries between spaces of weighted holomor-} phic functions on $\Delta$ with incomplete or non-radial weights. Let us determine the isometries of a space of weighted holomorphic functions where the weight is incomplete.

Proposition 27. Let $x_{0} \in(0,1)$ and suppose that $w:\left\{z:|z| \in\left[x_{0}, 1\right)\right\}$ $\rightarrow \mathbb{R}$ is a continuous strictly positive radial weight which converges to 0 as $|z|$ tends to 1 . Suppose that $w(x)$ is twice differentiable on $\left[x_{0}, 1\right)$ with $w^{\prime}(x)^{2}-w(x) w^{\prime \prime}(x)>0$ for $x \in\left[x_{0}, 1\right)$ and $w^{\prime}\left(x_{0}\right)<0$. Define $v: \Delta \rightarrow \mathbb{R}^{+}$ by

$$
v(z)= \begin{cases}w\left(x_{0}\right) & \text { if }|z|<x_{0}, \\ w(|z|) & \text { if }|z| \geq x_{0} .\end{cases}
$$

Then every isometry from $\mathcal{H}_{v_{0}}(\Delta)$ onto $\mathcal{H}_{v_{0}}(\Delta)$ is, modulo multiplication by $\lambda \in \Gamma$, composition with a rotation of $\Delta$.

Proof. It is shown in [8, Example 11] that $\mathcal{B}_{v}(\Delta)=\left\{z:|z| \in\left[x_{0}, 1\right)\right\}$. By the proof of the first Banach-Stone theorem we know that every isometry $T$ of $\mathcal{H}_{v_{0}}(\Delta)$ has the form

$$
T(f)(z)=h_{\phi}(z) f(\phi(z)) \quad \text { for all } f \in \mathcal{H}_{v_{0}}(\Delta), z \in \mathcal{B}_{v}(\Delta),
$$

where $\phi$ is a homeomorphism of $\mathcal{B}_{v}(\Delta)$ and $h_{\phi} \in \mathcal{H}_{v_{0}}(\Delta)$. We observe that $\phi$ must map $\check{\mathcal{B}}_{v}(\Delta)$ onto $\dot{\mathcal{B}}_{v}(\Delta)$ and $\mathcal{B}_{v}(\Delta) \backslash \check{\mathcal{B}}_{v}(\Delta)$ onto $\mathcal{B}_{v}(\Delta) \backslash \check{\mathcal{B}}_{v}(\Delta)$. Taking $f(z)=z$ and restricting $f$ to $\dot{\mathcal{B}}_{v}(\Delta)$ we see that $\phi$ is a biholomorphic mapping of $\dot{\mathcal{B}}_{v}(\Delta)$ onto itself. Since $\mathcal{B}_{v}(\Delta)=\left\{z: x_{0}<|z|<1\right\}$, [1, Proposition 1.1.32] implies that $\left.\phi\right|_{\mathcal{B}_{v}(\Delta)}$ is either $z \mapsto e^{i \theta} z$ or $z \mapsto x_{0} e^{i \theta} z^{-1}$. However, since $\left.\phi\right|_{\mathscr{B}_{v}(\Delta)}$ extends continuously to $\left\{z: x_{0} \leq|z|<1\right\}$ and $\phi$ maps $\mathcal{B}_{v}(\Delta) \backslash \dot{\mathcal{B}}_{v}(\Delta)$ onto $\mathcal{B}_{v}(\Delta) \backslash \dot{\mathcal{B}}_{v}(\Delta)$, the second possibility is ruled out and $\phi$ is a rotation. It now follows from Theorem 3 that $T$ is, modulo multiplication by $\lambda \in \Gamma$, composition with a rotation of $\Delta$.

Let us construct a weight with a finite isometry group.

ExAmple 28. Let $v(z):=\left(1-|z|^{2}\right)(1-x y)=v_{1,2}(z)(1-x y), z \in \Delta$. Then $\Lambda_{v}(\Delta)=\left\{R_{0}, R_{\pi}\right\}$. 
Proof. Let us first see that the $v$-boundary contains the diagonal $\{x-$ $i x: x \in(0,1)\}$. For $0<r<1$ let $\Gamma_{r}$ denote the circle with centre 0 and radius $r$. By the proof of [8, Proposition 11], given any function, $f$, of the form $f(z)=e^{\alpha e^{5 i \pi / 4} z}, \alpha>0, v_{1,2}(z)|f(z)|$ attains a unique maximum over $\Gamma_{r}$ at the intersection of $\Gamma_{r}$ and the diagonal $\{x-i x: x \in(0,1)\}$. The function $1-x y$ also attains its maximum over $\Gamma_{r}$ at the intersection $\Gamma_{r}$ and the diagonal $\{x-i x: x \in(-1,1)\}$. Hence $(1-x y) v_{1,2}(z)|f(z)|$ has a unique maximum over $\Gamma_{r}$ at the intersection of $\Gamma_{r}$ and the diagonal $\{x-i x: x \in(0,1)\}$. The restriction of the function $(1-x y) v_{1,2}(z)|f(z)|$ to the diagonal $\{x-i x: x \in(0,1)\}$ takes the form $\left(1-x^{2}-2 x^{4}\right)|f(x-i x)|$. Consider $x_{0}-i x_{0}$ with $x_{0} \in(0,1)$. An elementary calculation shows that we can choose $\alpha$ so that $\left(1-x^{2}-2 x^{4}\right)|f(z)|$ attains a unique maximum over $\{x-i x: x \in(0,1)\}$, and hence over $\Delta$, at $x_{0}-i x_{0}$. Thus each point of $\{x-i x: x \in(0,1)\}$ is a $v$-peak point and hence belongs to the $v$-boundary.

Let $\phi \in \Lambda_{v}(\Delta)$. Using Theorem 4 we see that each $\phi \in \Lambda_{v}(\Delta)$ is an automorphism of $\Delta$. If we operate in the same way as in previous examples, we get

$$
\triangle(\log \circ v)(z)=\triangle(\log v \circ \phi(z))
$$

Since

$$
\begin{aligned}
\triangle(\log \circ v)(z) & =\triangle\left(\log \circ v_{1,2}\right)(z)+\triangle \log (1-x y) \\
& =-\frac{4}{\left(1-|z|^{2}\right)^{2}}-\frac{|z|^{2}}{(1-x y)^{2}}
\end{aligned}
$$

we deduce that $\log \circ v$ is superharmonic. We have

$$
\triangle(\log v(\phi(z)))=\triangle(\log \circ v)(\phi(z))\left|\phi^{\prime}(z)\right|^{2},
$$

and letting $u_{1}(x, y)$ and $u_{2}(x, y)$ denote the real and imaginary part of $\phi$ respectively, we get

$$
\begin{aligned}
-\frac{4}{\left(1-|z|^{2}\right)^{2}}-\frac{|z|^{2}}{(1-x y)^{2}}=\left(-\frac{4}{\left(1-|\phi(z)|^{2}\right)^{2}}\right. & \\
& \left.\quad-\frac{|\phi(z)|^{2}}{\left(1-u_{1}(x, y) u_{2}(x, y)\right)^{2}}\right)\left|\phi^{\prime}(z)\right|^{2}
\end{aligned}
$$

on $\mathcal{B}_{v}(\Delta)$. Letting $z=0$ and using the fact that $\left|\phi^{\prime}(0)\right|=1-|\phi(0)|^{2}$ by the Schwarz-Pick theorem, we get

$$
4=4+\frac{|\phi(0)|^{2}\left(1-|\phi(0)|^{2}\right)^{2}}{\left(1-u_{1}(0,0) u_{2}(0,0)\right)^{2}}
$$

and thus $\phi(0)=0$. Therefore $\phi$ is a rotation.

First observe that $\left\{R_{0}, R_{\pi}\right\} \subset \Lambda_{v}(\Delta)$. We observe that the mapping $\mathcal{H}_{v_{0}}(\Delta) \rightarrow \mathcal{H}_{v_{0}}(\Delta), f \mapsto f$, is an isometry. So $R_{0} \in \Lambda_{v}(\Delta)$. Next consider 
the mapping $T_{\pi}: \mathcal{H}_{v_{0}}(\Delta) \rightarrow \mathcal{H}_{v_{0}}(\Delta), f \mapsto f \circ R_{\pi}$. We have

$$
\begin{aligned}
\left\|T_{\pi} f\right\|_{v} & =\left\|f \circ R_{\pi}\right\|_{v}=\sup _{z \in \Delta} v(z)\left|f \circ R_{\pi}(z)\right|=\sup _{z \in \Delta}(1-x y)\left(1-|z|^{2}\right)\left|f \circ R_{\pi}(z)\right| \\
& =\sup _{z \in \Delta}\left(1-R_{\pi}(x) R_{\pi}(y)\right)\left(1-\left|R_{\pi}(z)\right|^{2}\right)\left|f \circ R_{\pi}(z)\right|=\|f\|_{v} .
\end{aligned}
$$

Therefore $R_{\pi}$ is also in $\Lambda_{v}(\Delta)$.

By Corollary 25, if $R_{\theta} \in \Lambda_{v}(\Delta)$ then $\triangle \log v\left(e^{i \theta} z\right)=\triangle \log v(z)$ for all $z \in \mathcal{B}_{v}(\Delta)$. Since

$$
\triangle \log v\left(e^{i \theta} z\right)=\triangle \log v_{1,2}\left(e^{i \theta} z\right)-\frac{|z|^{2}}{(1-(x \cos \theta-y \sin \theta)(x \sin \theta+y \cos \theta))^{2}}
$$

and $\triangle \log v_{1,2}(z)$ is radial, we deduce that if $R_{\theta} \in \Lambda_{v}(\Delta)$ then

$$
\begin{aligned}
x y & =(x \cos \theta-y \sin \theta)(x \sin \theta+y \cos \theta) \\
& =x^{2} \sin \theta \cos \theta+x y\left(\cos ^{2} \theta-\sin ^{2} \theta\right)-y^{2} \sin \theta \cos \theta \\
& =\frac{x^{2}-y^{2}}{2} \sin (2 \theta)+x y \cos (2 \theta)
\end{aligned}
$$

for all $x+i y \in \mathcal{B}_{v}(\Delta)$. Since the diagonal $\{x-i x: x \in(0,1)\}$ is contained in $\mathcal{B}_{v}(\Delta)$ we get $\cos (2 \theta)=1$. This means that $\theta=k \pi, k \in \mathbb{Z}$.

Example 29. Define $v:(-1,1) \times(-1,1) \rightarrow \mathbb{R}$ by $v(z)=\left(1-x^{4}\right)\left(1-y^{4}\right)$. Then $\Lambda_{v}((-1,1) \times(-1,1))=\left\{\operatorname{Id}, R_{\pi / 2}, R_{\pi}, R_{3 \pi / 2}\right\}$.

Proof. By [8, Proposition 12] the weight $v$ is complete. By our first Banach-Stone theorem (Theorem 1) every isometry $T$ from $\mathcal{H}_{v_{0}}((-1,1) \times$ $(-1,1))$ onto $\mathcal{H}_{v_{0}}((-1,1) \times(-1,1))$ has the form

$$
T(f)(z)=h_{\phi}(z) f(\phi(z))
$$

for some automorphism $\phi$ of $(-1,1) \times(-1,1)$ and $h_{\phi} \in \mathcal{H}_{v_{0}}((-1,1) \times(-1,1))$. Furthermore,

$$
\left|h_{\phi}(z)\right|=\frac{v(\phi(z))}{v(z)} .
$$

Proceeding as before we get

$$
\triangle(\log v)(\phi(z))\left|\phi^{\prime}(z)\right|^{2}=\triangle(\log v)(z) .
$$

Since

$$
-\triangle\left(\log \left(1-x^{4}\right)\left(1-y^{4}\right)\right)=\frac{12 x^{2}}{1-x^{4}}+\frac{16 x^{6}}{\left(1-x^{4}\right)^{2}}+\frac{12 y^{2}}{1-y^{4}}+\frac{16 y^{6}}{\left(1-y^{4}\right)^{2}},
$$


writing $\phi(z)$ as $\phi(z)=u_{1}(x, y)+i u_{2}(x, y)$ we find that

$$
\begin{aligned}
\left(\left(\frac{\partial u_{1}}{\partial x}\right)^{2}+\left(\frac{\partial u_{2}}{\partial x}\right)^{2}\right) & \left(\frac{12 u_{1}(x, y)^{2}}{1-u_{1}(x, y)^{4}}+\frac{16 u_{1}(x, y)^{6}}{\left(1-u_{1}(x, y)^{4}\right)^{2}}\right. \\
& \left.+\frac{12 u_{2}(x, y)^{2}}{1-u_{2}(x, y)^{4}}+\frac{16 u_{2}(x, y)^{6}}{\left(1-u_{2}(x, y)^{4}\right)^{2}}\right) \\
& =\frac{12 x^{2}}{1-x^{4}}+\frac{16 x^{6}}{\left(1-x^{4}\right)^{2}}+\frac{12 y^{2}}{1-y^{4}}+\frac{16 y^{6}}{\left(1-y^{4}\right)^{2}} .
\end{aligned}
$$

Since $\left|\phi^{\prime}(z)\right| \neq 0$, setting $x=0, y=0$ we see that $u_{1}(0,0)=u_{2}(0,0)=0$. That is, $\phi(0)=0$. Applying [12, p. 17] we obtain $\left|\phi^{\prime}(0)\right|=1$.

Expanding $u_{1}(x, y)$ and $u_{2}(x, y)$ as a Taylor series in $x$ and $y$ about the origin, using the Cauchy-Riemann equations and the fact that $u_{1}$ and $u_{2}$ are harmonic we see that

$$
\begin{aligned}
& u_{1}(x, y)=a x+b y+c\left(x^{2}-y^{2}\right)-2 d x y+O(3), \\
& u_{2}(x, y)=-b x+a y+d\left(x^{2}-y^{2}\right)+2 c x y+O(3) .
\end{aligned}
$$

We claim that either $a$ or $b$ is 0 . Suppose that this is not true. Observe that $x^{3}$ and $y^{3}$ on the left-hand side of (1) can only occur when $\left(\partial u_{1} / \partial x\right)^{2}$ and $\left(\partial u_{1} / \partial y\right)^{2}$ are multiplied by $12 u_{1}(x, y)^{2}$ and $12 u_{2}(x, y)^{2}$. Equating powers of $x^{3}$ and $y^{3}$ on both sides we get

$$
a c-b d=0, \quad-a d-b c=0 .
$$

Considering these as linear equations in $c$ in $d$, since $-a^{2}-b^{2} \neq 0$, we deduce that $c$ and $d$ are 0 . This gives

$$
\begin{aligned}
& u_{1}(x, y)=a x+b y+e x^{3}+3 f x^{2} y-3 e x y^{2}-f y^{3}+O(4), \\
& u_{2}(x, y)=-b x+a y-f x^{3}+3 e x^{2} y+3 f x y^{2}-e y^{3}+O(4) .
\end{aligned}
$$

Equating coefficients of $x^{4}$ (or $y^{4}$ ) and $x^{3} y$ (or $x y^{3}$ ) in (1) as above we see that

$$
a e+b f=0, \quad a f-b e=0 .
$$

As before, this shows that both $e$ and $f$ are 0 .

It follows that the terms which involve $x^{6}$ and $y^{6}$ on the left-hand side of (1) can only arise from $u_{1}(x, y)^{6}$ and $u_{2}(x, y)^{6}$. But comparing coefficients of $x^{6}$ and $y^{6}$ we get $a^{6}+b^{6}=1$. Since $a^{2}+b^{2}=1$ and $a$ and $b$ are both non-zero, this is impossible. Hence either $a$ or $b$ is 0 .

Suppose that $b=0$. Then $a= \pm 1$. We claim that $u_{1}(x, y)= \pm x$ and $u_{2}(x, y)= \pm y$. (If $a=0$, a similar argument will show that $u_{1}(x, y)= \pm y$ and $u_{2}(x, y)=\mp x$.) Suppose that this is not true. Suppose $u_{1}(x, y)$ or $u_{2}(x, y)$ contains terms of the form $a x^{k}$ for $k \geq 2$. Let $k$ be the smallest 
integer with this property. Putting this into (1) we obtain

$$
\begin{aligned}
& 12 x^{2} \sum_{n=0}^{\infty} x^{4 n}+16 x^{6} \sum_{n=1}^{\infty} n x^{4(n-1)}+12 y^{2} \sum_{n=0}^{\infty} y^{4 n} \\
& \quad+16 y^{6} \sum_{n=1}^{\infty} n y^{4(n-1)}+a^{4} x^{4 k}+O(6 k) \\
& =12 x^{2} \sum_{n=0}^{\infty} x^{4 n}+16 x^{6} \sum_{n=1}^{\infty} n x^{4(n-1)}+12 y^{2} \sum_{n=0}^{\infty} y^{4 n}+16 y^{6} \sum_{n=1}^{\infty} n y^{4(n-1)} .
\end{aligned}
$$

So $u_{1}(x, y)$ or $u_{2}(x, y)$ cannot contain terms of this form $a x^{k}$. An analogous argument shows that $u_{1}(x, y)$ or $u_{2}(x, y)$ cannot contain a term of the form $a y^{k}$.

Finally, suppose that $u_{1}(x, y)$ contains a term of the form $a x^{n} y^{m}$ for $n, m>0$. Let $m$ be the smallest positive integer with this property. Since $u_{1}(x, y)$ and $u_{2}(x, y)$ satisfy the Cauchy-Riemann equations it follows that $u_{2}(x, y)$ contains a term of the form $b x^{n+1} y^{m-1}$. If $m-1=0$ this would contradict the fact that $u_{2}(x, y)$ does not contain a term of the form $b x^{k}$ for any $k>1$. Hence $m \geq 2$. Then $\partial u_{2} / \partial y$ contains a term of the form $b x^{n+1} y^{m-2}$, and therefore by the Cauchy-Riemann equations again, $u_{1}(x, y)$ contains a term of the form $c x^{n+2} y^{m-2}$. If $m=2$ this contradicts the previous paragraph, while if $m>2$ this contradicts our choice of $m$. Hence we have four possibilities:

$$
\begin{array}{ll}
u_{1}(x, y)=x, & u_{2}(x, y)=y, \\
u_{1}(x, y)=-x, & u_{2}(x, y)=-y, \\
u_{1}(x, y)=y, & u_{2}(x, y)=-x, \\
u_{1}(x, y)=-y, & u_{2}(x, y)=x .
\end{array}
$$

These give the identity and the rotations by $\pi, \pi / 2$ and $3 \pi / 2$ respectively.

COROLlary 30. There is a weight $w$ on the open unit disc $\Delta$ with $\Lambda_{w}(\Delta)=\left\{\mathrm{Id}, R_{\pi / 2}, R_{\pi}, R_{3 \pi / 2}\right\}$.

Proof. By the Riemann mapping theorem, $\Delta$ and $(-1,1) \times(-1,1)$ are conformally equivalent. In fact, since $\operatorname{Aut}(\Delta)$ acts transitively on $\Delta$, we can assume that there is a conformal mapping $\phi: \Delta \rightarrow(-1,1) \times(-1,1)$ such that $\phi(0)=0$. If we set $w(z)=\left(1-(\operatorname{Re} \phi(z))^{4}\right)\left(1-(\operatorname{Im} \phi(z))^{4}\right)$, Example 29 gives us a weight $w$ with four elements. Since $\phi(0)=0$ each of these elements is a rotation and hence $\Lambda_{w}(\Delta)=\left\{\mathrm{Id}, R_{\pi / 2}, R_{\pi}, R_{3 \pi / 2}\right\}$.

10. Isometries of weighted spaces of holomorphic functions on polydisc domains. Let us now consider holomorphic functions on balanced domains of the form $U \times V$ with radial Cartesian weights, that is, 
weights of the form $v \times w$ where $v$ is a radial weight on $U$ and $w$ is a radial weight on $V$ which converge to 0 on their respective boundaries. We have previously seen in $\left[8\right.$, Proposition 20] that $\mathcal{B}_{v \times w}(U \times V)=\mathcal{B}_{v}(U) \times \mathcal{B}_{w}(V)$. We shall use $S_{2}$ to denote the permutation group of order 2 .

THEOREM 31. Let $U$ and $V$ be balanced bounded open subsets of $\mathbb{C}^{n}$ and $\mathbb{C}^{m}$ respectively, and let $v$ and $w$ be continuous strictly positive radial weights on $U$ and $V$ respectively which converge to zero on their respective boundaries. Assume that both $\mathcal{B}_{v}(U)$ and $\mathcal{B}_{w}(V)$ have non-empty interiors and that $U$ (resp. $V)$ contains no positive-dimensional analytic set in an open subset of $\mathbb{C}^{n}$ (resp. $\left.\mathbb{C}^{m}\right)$.

(a) If $\mathcal{H}_{v_{0}}(U)$ is not isometrically isomorphic to $\mathcal{H}_{w_{0}}(V)$ then every isometry $T$ of the space $\mathcal{H}_{(v \times w)_{0}}(U \times V)$ has the form

$$
T(f)\left(z_{1}, z_{2}\right)=h_{\phi}\left(z_{1}\right) h_{\psi}\left(z_{2}\right) f\left(\phi\left(z_{1}\right), \psi\left(z_{2}\right)\right)
$$

for all $\left(z_{1}, z_{2}\right) \in \dot{\mathcal{B}}_{v \times w}(U \times V)$, where $\phi \in \Lambda_{v}(U), \psi \in \Lambda_{w}(V), h_{\phi} \in$ $\mathcal{H}_{v_{0}}(U)$ and $h_{\psi} \in \mathcal{H}_{w_{0}}(V)$.

(b) If $\mathcal{H}_{v_{0}}(U)$ is isometrically isomorphic to $\mathcal{H}_{w_{0}}(V)$ then every isometry $T$ of the space $\mathcal{H}_{(v \times w)_{0}}(U \times V)$ has either the form

$$
T(f)\left(z_{1}, z_{2}\right)=h_{\phi}\left(z_{1}\right) h_{\psi}\left(z_{2}\right) f\left(\phi\left(z_{1}\right), \psi\left(z_{2}\right)\right)
$$

for all $\left(z_{1}, z_{2}\right) \in \dot{\mathcal{B}}_{v \times w}(U \times V)$, where $\phi \in \Lambda_{v}(U), \psi \in \Lambda_{w}(V), h_{\phi} \in$ $\mathcal{H}_{v_{0}}(U)$ and $h_{\psi} \in \mathcal{H}_{w_{0}}(V)$, or the form

$$
T(f)\left(z_{1}, z_{2}\right)=h_{\phi}\left(\eta\left(z_{2}\right)\right) h_{\psi}\left(\eta^{-1}\left(z_{1}\right)\right) f\left(\phi\left(\eta\left(z_{2}\right)\right), \psi\left(\eta^{-1}\left(z_{1}\right)\right)\right)
$$

for all $\left(z_{1}, z_{2}\right) \in \dot{\mathcal{B}}_{v \times w}(U \times V)$, for some biholomorphic mapping $\eta: \grave{\mathcal{B}}_{w}(V) \rightarrow \dot{\mathcal{B}}_{v}(U)$, where $\phi \in \Lambda_{v}(U), \psi \in \Lambda_{w}(V), h_{\phi} \in \mathcal{H}_{v_{0}}(U)$ and $h_{\psi} \in \mathcal{H}_{w_{0}}(V)$.

Proof. By the first Banach-Stone theorem (Theorem 1) we deduce that every isometry $T$ of $\mathcal{H}_{(v \times w)_{0}}(U \times V)$ has the form

$$
T(f)\left(z_{1}, z_{2}\right)=h_{\phi}\left(z_{1}, z_{2}\right) f\left(\phi\left(z_{1}, z_{2}\right)\right)
$$

where $\phi$ is an automorphism of $\dot{\mathcal{B}}_{v \times w}(U \times V)=\dot{\mathcal{B}}_{v}(U) \times \dot{\mathcal{B}}_{w}(V)$ and $h_{\phi} \in$ $\mathcal{H}_{(v \times w)_{0}}(U \times V)$. When $\mathcal{H}_{v_{0}}(U)$ is not isometrically isomorphic to $\mathcal{H}_{w_{0}}(V)$, applying [27, Proposition 5.I] (see also [10]) we see such $\phi$ has the form

$$
\phi\left(z_{1}, z_{2}\right)=\left(\phi_{1}\left(z_{1}\right), \phi_{2}\left(z_{2}\right)\right)
$$

where $\phi_{1}$ (resp. $\left.\phi_{2}\right)$ is an automorphism of $\dot{\mathcal{B}}_{v}(U)$ (resp. $\left.\stackrel{\circ}{\mathcal{B}}_{w}(V)\right)$. Furthermore, the first Banach-Stone theorem tells us that

$$
\left|h_{\phi}\left(z_{1}, z_{2}\right)\right|=\frac{v\left(\phi_{1}\left(z_{1}\right)\right)}{v\left(z_{1}\right)} \frac{w\left(\phi_{2}\left(z_{2}\right)\right)}{w\left(z_{2}\right)} .
$$

The transpose, $T^{*}$, of $T$ will give an isometry of $\mathcal{H}_{(v \times w)_{0}}(U \times V)^{\prime}$. In [8, Corollary 19] we observed that $\mathcal{H}_{(v \times w)_{0}}(U \times V)^{\prime}$ is isometrically isomorphic 
to $\mathcal{H}_{v_{0}}(U)^{\prime} \widehat{\otimes}_{\pi} \mathcal{H}_{w_{0}}(V)^{\prime}$ and under this isometry $v(x) w(y) \delta_{(x, y)}$ is mapped to $v(x) \delta_{x} \otimes w(y) \delta_{y}$. Suppose $(x, y) \in \stackrel{\circ}{\mathcal{B}}_{v \times w}(U \times V)=\dot{\mathcal{B}}_{v}(U) \times \stackrel{\circ}{\mathcal{B}}_{w}(V)$. The proof of the first Banach-Stone theorem tells us that there is $\alpha\left(z_{1}, z_{2}\right) \in \Gamma$ with

(*) $\quad T^{*}\left(v\left(z_{1}\right) \delta_{z_{1}} \otimes w\left(z_{2}\right) \delta_{z_{2}}\right)=\alpha\left(z_{1}, z_{2}\right) v\left(\phi_{1}\left(z_{1}\right)\right) \delta_{\phi_{1}\left(z_{1}\right)} \otimes w\left(\phi_{2}\left(z_{2}\right)\right) \delta_{\phi_{2}\left(z_{2}\right)}$.

As $\left(T^{*}\right)^{-1} \operatorname{maps} \alpha\left(z_{1}, z_{2}\right) v\left(\phi_{1}\left(z_{1}\right)\right) \delta_{\phi_{1}\left(z_{1}\right)} \otimes w\left(\phi_{2}\left(z_{2}\right)\right) \delta_{\phi_{2}\left(z_{2}\right)}$ onto $v\left(z_{1}\right) \delta_{z_{1}}$ $\otimes w\left(z_{2}\right) \delta_{z_{2}}$, we see that $\left.T^{*}\right|_{B_{\mathcal{H}_{0}(U)^{\prime}}}$ maps $B_{\mathcal{H}_{v_{0}}(U)^{\prime}}$ onto $B_{\mathcal{H}_{v_{0}}(U)^{\prime}}$, and $\left.T^{*}\right|_{B_{\mathcal{H}_{w_{0}}(V)^{\prime}}}$ maps $B_{\mathcal{H}_{w_{0}}(V)^{\prime}}$ onto $B_{\mathcal{H}_{w_{0}}(V)^{\prime}}$. Therefore, $\phi_{1} \in \Lambda_{v}(U)$ and $\phi_{2} \in$ $\Lambda_{w}(V)$. From $(*)$ we now get $\left|h_{\phi}\left(z_{1}, z_{2}\right)\right|=\left|h_{\phi_{1}}\left(z_{1}\right) h_{\phi_{2}}\left(z_{2}\right)\right|$ for all $\left(z_{1}, z_{2}\right) \in$ $\stackrel{\mathcal{B}}{v \times w}(U \times V)$ and hence by the open mapping theorem we have $h_{\phi}=\lambda h_{\phi_{1}} h_{\phi_{2}}$ for some $\lambda \in \Gamma$.

The second case is almost identical to the first when we allow for the fact that there are automorphisms $\phi$ of $\dot{\mathcal{B}}_{v \times w}(U \times V)$ of the form

$$
\phi\left(z_{1}, z_{2}\right)=\left(\phi_{1}\left(\eta\left(z_{2}\right)\right), \phi_{2}\left(\eta^{-1}\left(z_{1}\right)\right)\right)
$$

for some automorphisms $\phi_{1}, \phi_{2}$ of $\dot{\mathcal{B}}_{v}(U)$ (or equivalently $\dot{\mathcal{B}}_{w}(V)$ ) and $\eta$ is a biholomorphic mapping from $\dot{\mathcal{B}}_{w}(V)$ onto $\dot{\mathcal{B}}_{v}(U)$ (see $[27$, Proposition 5.I] or [10]).

Corollary 32. Let $U$ and $V$ be balanced bounded open subsets of $\mathbb{C}^{n}$ and $\mathbb{C}^{m}$ respectively, and let $v$ and $w$ be continuous strictly positive radial weights on $U$ and $V$ respectively which converge to zero on their respective boundaries. Assume that both $\mathcal{B}_{v}(U)$ and $\mathcal{B}_{w}(V)$ have non-empty interior and that $U$ (resp. $V)$ contains no positive-dimensional analytic set in an open subset of $\mathbb{C}^{n}$ (resp. $\left.\mathbb{C}^{m}\right)$.

(a) If $\mathcal{H}_{v_{0}}(U)$ is not isometrically isomorphic to $\mathcal{H}_{w_{0}}(V)$ then

$$
\Lambda_{v \times w}(U \times V)=\Lambda_{v}(U) \times \Lambda_{w}(V) \text { and } \pi_{v \times w}=\pi_{v} \times \pi_{w} .
$$

(b) If $\mathcal{H}_{v_{0}}(U)$ is isometrically isomorphic to $\mathcal{H}_{w_{0}}(V)$ then

$$
\Lambda_{v \times w}(U \times V)=\Lambda_{v}(U) \times \Lambda_{w}(V) \quad \text { modulo } S_{2}
$$

and

$$
\pi_{v \times w}=\pi_{v} \times \pi_{w} \quad \text { modulo } S_{2} .
$$

We list some of the isometries that Theorem 31 allows us to classify.

EXAMPLE 33.

(a) Every isometry $T$ of $\mathcal{H}_{\left(v_{\alpha, 2} \times v_{\alpha, 2}\right)_{0}}(\Delta \times \Delta)$ has the form

$$
T(f)\left(z_{1}, z_{2}\right)=\left(\phi_{1}^{\prime}\left(z_{\sigma(1)}\right) \phi_{2}^{\prime}\left(z_{\sigma(2)}\right)\right)^{\alpha} f\left(\phi_{1}\left(z_{\sigma(1)}\right), \phi_{2}\left(z_{\sigma(2)}\right)\right)
$$

where $\phi_{1}, \phi_{2}$ are in $\operatorname{Aut}(\Delta)$ and $\sigma \in S_{2}$.

(b) For $\beta \neq 2$, every isometry $T$ of $\mathcal{H}_{\left(v_{\alpha, \beta} \times v_{\alpha, \beta}\right)_{0}}(\Delta \times \Delta)$ has the form

$$
T(f)\left(z_{1}, z_{2}\right)=\lambda f\left(e^{i \theta_{1}}\left(z_{\sigma(1)}\right), e^{i \theta_{2}}\left(z_{\sigma(2)}\right)\right)
$$

where $e^{i \theta_{1}}, e^{i \theta_{2}}$ and $\lambda$ are in $\Gamma$ and $\sigma \in S_{2}$. 
(c) For $\beta, \beta^{\prime} \neq 2$ and distinct, every isometry $T$ of $\mathcal{H}_{\left(v_{\alpha, \beta} \times v_{\alpha, \beta^{\prime}}\right)_{0}}(\Delta \times \Delta)$ has the form

$$
T(f)\left(z_{1}, z_{2}\right)=\lambda f\left(e^{i \theta_{1}} z_{1}, e^{i \theta_{2}} z_{2}\right)
$$

where $\theta_{1}, \theta_{2}$ and $\lambda$ are in $\Gamma$.

Acknowledgements. The authors wish to thank the referee for his/her useful comments and suggestions.

During the summers of 2001, 2002 and 2003 the first author made short visits to the University of Valencia. He wishes to thank the Department of Mathematical Analysis there for its hospitality during those visits.

The second author was supported by MEC and FEDER Project MTM2005-08210.

\section{References}

[1] M. Abate, Iteration Theory of Holomorphic Maps on Taut Manifolds, Mediterranean Press, 1989.

[2] J. Araujo and J. Font, Linear isometries between subspaces of continuous functions, Trans. Amer. Math. Soc. 349 (1997), 413-428.

[3] E. Behrends, M-Structure and the Banach-Stone Theorem, Lecture Notes in Math. 736, Springer, Berlin, 1979.

[4] K. D. Bierstedt, J. Bonet and A. Galbis, Weighted spaces of holomorphic functions on balanced domains, Michigan Math. J. 40 (1993), 271-297.

[5] J. Bonet, P. Domański, M. Lindström and J. Taskinen, Composition operators between weighted Banach spaces of analytic functions, J. Austral. Math. Soc. Ser. A 64 (1998), 101-118.

[6] J. Bonet and E. Wolf, A note on weighted Banach spaces of holomorphic functions, Arch. Math. (Basel) 81 (2003), 650-654.

[7] C. Boyd and P. Rueda, The v-boundary of weighted spaces of holomorphic functions, Ann. Acad. Sci. Fenn. Math. 30 (2005), 337-352.

[8] - - - Complete weights and v-peak points of spaces of weighted holomorphic functions, Israel J. Math. 155 (2006), 57-80.

[9] - - - Bergman and Reinhardt weighted spaces of holomorphic functions, Illinois J. Math. 49 (2005), 217-236.

[10] H. Cartan, Sur les fonctions de $n$ variables complexes : Les transformations du produit topologique de deux domaines bornés, Bull. Soc. Math. France 64 (1936), $37-48$.

[11] J. A. Cima and W. R. Wogen, On isometries of the Bloch space, Illinois J. Math. 24 (1980), 313-316.

[12] S. Dineen, The Schwarz Lemma, Oxford Math. Monogr., Clarendon Press, Oxford, 1989.

[13] F. Forelli, The isometries of $H^{p}$, Canad. J. Math. 16 (1964), 721-728.

[14] P. Harmand and Å. Lima, Banach spaces which are M-ideals in their biduals, Trans. Amer. Math. Soc. 283 (1984), 253-264.

[15] P. Harmand, D. Werner and W. Werner, M-Ideals in Banach Spaces and Banach Algebras, Lecture Notes in Math. 1547, Springer, Berlin, 1993. 
[16] K. Jarosz, Isometries between injective tensor products of Banach spaces, Pacific J. Math. 121 (1986), 383-396.

[17] M. Klimek, Pluripotential Theory, London Math. Soc. Monogr., Oxford Univ. Press, Oxford, 1991.

[18] C. J. Kolaski, Isometries of Bergman spaces over bounded Runge domains, Canad. J. Math. 33 (1981), 1157-1164.

[19] —, Surjective isometries of weighted Bergman spaces, Proc. Amer. Math. Soc. 105 (1989), 652-657.

[20] W. Lusky, On the structure of $\mathcal{H}_{v_{0}}(D)$ and $h_{v_{0}}(D)$, Math. Nachr. 159 (1992), 279289.

[21] —, On weighted spaces of harmonic and holomorphic functions, J. London Math. Soc. 51 (1995), 309-320.

[22] —, On generalized Bergman spaces, Studia Math. 119 (1996), 77-95.

[23] - On the isomorphic classification of weighted spaces of holomorphic functions, Acta Univ. Carolin. Math. Phys. 41 (2000), 51-60.

[24] - On the Fourier series of unbounded harmonic functions, J. London Math. Soc. 61 (2000), 568-580.

[25] - On the isomorphism classes of some spaces of harmonic and holomorphic functions, preprint.

[26] M. Nagasawa, Isomorphisms between commutative Banach algebras with an application to rings of analytic functions, Kōdai Math. Sem. Rep. 11 (1959), 182-188.

[27] R. Narasimhan, Several Complex Variables, Chicago Lectures in Math., Univ. of Chicago Press, Chicago, 1971.

[28] T. Ransford, Potential Theory in the Complex Plane, London Math. Soc. Student Texts 28, Cambridge Univ. Press, Cambridge, 1995.

[29] W. Rudin, $L^{p}$-isometries and equimeasurability, Indiana Univ. Math. J. 25 (1976), $215-228$.

[30] - Function Theory in the Unit Ball of $\mathbb{C}^{n}$, Grundlehren Math. Wiss. 241, Springer, New York, 1980.

[31] W. M. Ruess and C. P. Stegall, Extreme points in duals of operator spaces, Math. Ann. 261 (1982), 535-546.

[32] E. Thorp and R. Whitley, The strong maximum modulus theorem for analytic functions into a Banach space, Proc. Amer. Math. Soc. 18 (1967), 640-646.

School of Mathematical Sciences

University College Dublin

Belfield, Dublin 4, Ireland

E-mail: Christopher.Boyd@ucd.ie
Departamento de Análisis Matemático Facultad de Matemáticas Universidad de Valencia 46100 Burjasot, Valencia, Spain E-mail: Pilar.Rueda@uv.es

Received April 30, 2007

Revised version Jully 22, 2008 